\title{
Synthesis of 4-Arylpiperidines from 1-Benzyl-4-Piperidone: Application of the Shapiro Reaction and Alkenylsilane Cross-Coupling
}

\author{
Christie Morrill* and Neelakandha S. Mani \\ Department of Drug Discovery, Johnson \& Johnson Pharmaceutical R\&D LLC \\ 3210 Merryfield Row, San Diego, California 92121 \\ CMorril3@prdus.jnj.com
}

\section{Supporting Information}

S-2: $\quad$ General experimental methods

$S-2$ - S-5: $\quad$ Experimental procedures

S-2 - S-9: $\quad$ Characterization data $\left({ }^{1} \mathrm{H}\right.$ NMR, ${ }^{13} \mathrm{C}$ NMR, and HRMS) for compounds 2 , 4, 6-7, and all compounds reported in Tables 2 and 3

S-10 - S-35: $\quad{ }^{1} \mathrm{H}$ and ${ }^{13} \mathrm{C}$ NMR spectra for compounds $2,4,6$-7, and all compounds reported in Tables 2 and 3 


\section{General experimental methods:}

${ }^{1} \mathrm{H}$ and ${ }^{13} \mathrm{C}$ NMR spectra were recorded on Bruker 400 or $500 \mathrm{MHz}$ NMR spectrometers. Flash column chromatography was performed on CombiFlash Companion systems (Isco, Inc.) with RediSep pre-packed silica gel cartridges. HRMS (ESI) was performed on a Bruker $\mu$ Tof. Elemental analysis was performed by NuMega Resonance Labs, Inc. (San Diego, CA 92121). All commercially available reagents were purchased and used without further purification.

\section{Experimental procedures:}

\section{$N$-(1-Benzyl-piperidin-4-ylidene)- $N$ '-tosyl-hydrazine (2)}<smiles>Cc1ccc(S(=O)(=O)NN=C2CCN(Cc3ccccc3)CC2)cc1</smiles>

To a 2-L round-bottomed flask, added $86 \mathrm{~g}$ (462 mmol) $p$-toluene sulfonhydrazide (1) and $1 \mathrm{~L}$ ethanol, then placed under a nitrogen atmosphere. [Reaction formed a white suspension.] With mechanical stirring, added $75 \mathrm{~mL}$ (420 mmol) 1-benzyl-4-piperidone. [Reaction quickly became almost completely clear, and after about 15 minutes, 2 began to precipitate.] Let stir at room temperature for a total of 3.5 hours, then collected the precipitate via filtration, rinsing the filter cake with cold ethanol. Let the filter cake dry under vacuum (room temperature, ca. 0.2 torr) for a few hours, then ground the solid into a fine powder with mortar and pestle and placed under vacuum overnight (room temperature, ca. 0.2 torr). Obtained $111 \mathrm{~g}$ of 2 as a crystalline, white solid $(74 \%,>95 \%$ purity by ${ }^{1} \mathrm{H}$ NMR). This material was used in subsequent reactions without further purification. ${ }^{1} \mathrm{H}$ NMR $\left(500 \mathrm{MHz}, \mathrm{CDCl}_{3}\right): \delta=7.82(2 \mathrm{H}, \mathrm{d}, J=8.3 \mathrm{~Hz}), 7.27(7 \mathrm{H}, \mathrm{m})$, $3.49(2 \mathrm{H}, \mathrm{s}), 2.51(2 \mathrm{H}, \mathrm{t}, J=5.9 \mathrm{~Hz}), 2.46(2 \mathrm{H}, \mathrm{t}, J=6.0 \mathrm{~Hz}), 2.42(3 \mathrm{H}, \mathrm{s}), 2.34(4 \mathrm{H}, \mathrm{m})$. ${ }^{13} \mathrm{C} \mathrm{NMR}\left(125 \mathrm{MHz}, \mathrm{CDCl}_{3}\right): \delta=159.6,143.9,138.0,135.4,129.5,128.9,128.3,128.1$, 127.2, 62.3, 53.2, 51.8, 34.5, 26.8, 21.6. HRMS: $\mathrm{M}+\mathrm{H}$ calculated for $\mathrm{C}_{19} \mathrm{H}_{23} \mathrm{~N}_{3} \mathrm{O}_{2} \mathrm{~S}=$ 358.1584 ; observed $\mathrm{m} / \mathrm{z}=358.1595(-3.1 \mathrm{ppm})$. Melting point $=159-161{ }^{\circ} \mathrm{C}$. [Material decomposed to form an orange solid around $150{ }^{\circ} \mathrm{C}$.]

\section{1-Benzyl-4-(benzyldimethylsilanyl)-1,2,3,6-tetrahydro-pyridine (4)}<smiles>C[Si](C)(Cc1ccccc1)C1=CCN(Cc2ccccc2)CC1</smiles>

To a 1-L round-bottomed flask, added $18.6 \mathrm{~g}(52 \mathrm{mmol}) 2$ and $260 \mathrm{~mL}$ hexanes. Placed under a nitrogen atmosphere, added $40 \mathrm{~mL}$ ( $267 \mathrm{mmol})$ TMEDA, placed in a dry ice/acetone bath, and let stir for about 25 minutes. [Reaction formed a white suspension.] Via addition funnel, added $160 \mathrm{~mL}(256 \mathrm{mmol}) n$-butyllithium solution $(1.6 \mathrm{M}$ in 
hexanes) dropwise over about 20 minutes. [Reaction turned into a yellow-orange suspension, and the internal temperature rose by about $10{ }^{\circ} \mathrm{C}$.] Let stir at $-78{ }^{\circ} \mathrm{C}$ for 15 minutes after all of the $n$-butyllithium solution had been added, then removed flask from the dry ice bath and let stir for an additional 1.5 hours. [The internal temperature reached room temperature (ca. $18{ }^{\circ} \mathrm{C}$ ) over the course of about 1 hour. Over this time period, bubbling was observed, and the reaction slowly became reddish-orange and then reddishbrown.] Next, placed reaction in an ice bath, let stir for about 10 minutes, then added 14 $\mathrm{mL}$ (77 mmol) benzyldimethylsilyl chloride (neat) via syringe over about 1 minute. [Reaction quickly turned more reddish in color, and the internal temperature rose by about $15{ }^{\circ} \mathrm{C}$.] Let reaction stir overnight (ca. 16 hours), without maintaining the ice bath. The following day, placed reaction in an ice bath, let stir for about 15 minutes, and then slowly added $400 \mathrm{~mL}$ water, portionwise at first. [Reaction turned from reddish-brown to bright yellow, and the internal temperature rose by about $20^{\circ} \mathrm{C}$.] Transferred to a separatory funnel, separated the aqueous and organic layers, and extracted the aqueous layer two times with $300 \mathrm{~mL}$ hexanes. [The aqueous and organic layers did not separate immediately, but clean separation was achieved by letting the mixture sit undisturbed for 10-15 minutes.] Dried the combined organic layers with $\mathrm{Na}_{2} \mathrm{SO}_{4}$, concentrated in vacuo, and purified the crude material by silica gel chromatography (automated column, $330 \mathrm{~g}$ $\mathrm{SiO}_{2}, 0 \%$ to $20 \%$ EtOAc in hexanes gradient). Obtained $12.19 \mathrm{~g}$ of $\mathbf{4}$ as an orange oil (73\%). ${ }^{1} \mathrm{H}$ NMR $\left(500 \mathrm{MHz}, \mathrm{CDCl}_{3}\right): \delta=7.32(5 \mathrm{H}, \mathrm{m}), 7.21(2 \mathrm{H}, \mathrm{t}, J=7.6 \mathrm{~Hz}), 7.08$ $(1 \mathrm{H}, \mathrm{t}, J=7.4 \mathrm{~Hz}), 7.00(2 \mathrm{H}, \mathrm{d}, J=7.0 \mathrm{~Hz}), 5.93(1 \mathrm{H}, \mathrm{m}), 3.59(2 \mathrm{H}, \mathrm{s}), 3.03(2 \mathrm{H}, \mathrm{m})$, $2.53(2 \mathrm{H}, \mathrm{t}, J=5.6 \mathrm{~Hz}), 2.15(2 \mathrm{H}, \mathrm{m}), 2.15(2 \mathrm{H}, \mathrm{s}), 0.036(6 \mathrm{H}, \mathrm{s}) .{ }^{13} \mathrm{C} \mathrm{NMR}(100 \mathrm{MHz}$, $\left.\mathrm{CDCl}_{3}\right): \delta=140.1,138.2,135.2,134.8,129.2,128.18,128.17,128.0,127.0,123.9,63.0$, 54.3, 49.7, 28.0, 25.1, -4.5. HRMS: $\mathrm{M}+\mathrm{H}$ calculated for $\mathrm{C}_{21} \mathrm{H}_{27} \mathrm{NSi}=322.1986$; observed $\mathrm{m} / \mathrm{z}=322.2000(-4.5 \mathrm{ppm})$.

\section{1-Benzyl-4-(benzyldimethylsilanyl)-1,2,3,6-tetrahydro-pyridine hydrochloride (6)}

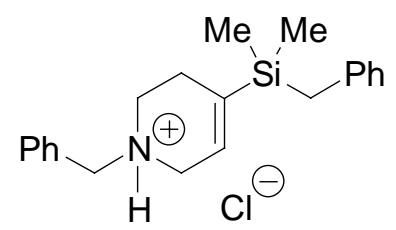

To a 250-mL Erlenmeyer flask, open to air, added $10.81 \mathrm{~g}(33.6 \mathrm{mmol}) 4$ and $70 \mathrm{~mL}$ isopropanol. Let stir at room temperature for about 5 minutes, then added $4.2 \mathrm{~mL}(50.4$ mmol) concentrated $\mathrm{HCl}$. [No immediate precipitation was observed.] Let stir at room temperature overnight. The following day, [A large amount of precipitate was observed at this time.] placed in an ice bath, let stir for approximately 2 hours, then filtered, rinsing the filter cake with approximately $15 \mathrm{~mL}$ of cold isopropanol. Obtained $7.85 \mathrm{~g}$ of 6 as a white, crystalline solid (65\%). [Upon treatment of the mother liquor with $3 \mathrm{M} \mathrm{NaOH}$ solution, followed by column chromatography, $2.56 \mathrm{~g}$ (7.96 mmol) 4 was recovered.] For 6: ${ }^{1} \mathrm{H}$ NMR $\left(500 \mathrm{MHz}, \mathrm{CDCl}_{3}\right): \delta=12.61(1 \mathrm{H}, \mathrm{s}), 7.63(2 \mathrm{H}, \mathrm{m}), 7.40(3 \mathrm{H}, \mathrm{m}), 7.18$ $(2 \mathrm{H}, \mathrm{t}, J=7.6 \mathrm{~Hz}), 7.05(1 \mathrm{H}, \mathrm{t}, J=7.4 \mathrm{~Hz}), 6.96(2 \mathrm{H}, \mathrm{d}, J=7.1 \mathrm{~Hz}), 5.75(1 \mathrm{H}, \mathrm{m}), 4.18$ $(1 \mathrm{H}, \mathrm{dd}, J=13.0,5.4 \mathrm{~Hz}), 4.08(1 \mathrm{H}, \mathrm{dd}, J=13.0,5.8 \mathrm{~Hz}), 3.74(1 \mathrm{H}, \mathrm{d}, J=17.0 \mathrm{~Hz}), 3.32$ 
(2H, m), $2.83(1 \mathrm{H}, \mathrm{m}), 2.67(1 \mathrm{H}, \mathrm{m}), 2.14(2 \mathrm{H}, \mathrm{s}), 2.12(1 \mathrm{H}, \mathrm{m}), 0.085(3 \mathrm{H}, \mathrm{s}), 0.080$ $(3 \mathrm{H}, \mathrm{s}) .{ }^{13} \mathrm{C} \mathrm{NMR}\left(125 \mathrm{MHz}, \mathrm{CDCl}_{3}\right): \delta=139.0,137.4,131.2,130.0,129.2,128.4$, 128.3, 128.0, 126.8, 124.4, 58.2, 49.2, 47.3, 24.8, 23.2, -4.50, -4.54. HRMS: M+H calculated for $\mathrm{C}_{21} \mathrm{H}_{28} \mathrm{NSi}=323.2064$; observed $\mathrm{m} / \mathrm{z}=323.2079$ (-4.7 ppm). Elemental analysis: calculated: C: $70.46 \%, \mathrm{H}: 7.88 \%, \mathrm{~N}: 3.91 \%$; observed: $\mathrm{C}: 70.74 \%(+0.28 \%)$, H: $8.24 \%(+0.36 \%), \mathrm{N}: 4.08 \%(+0.17 \%)$. Melting point $=173-175{ }^{\circ} \mathrm{C}$. [Possible decomposition observed at melting point temperature.]

\section{4-(Benzyldimethylsilanyl)-3,6-dihydro-2H-pyridine-1-t-butoxycarbonyl (7)}<smiles>CC(C)(C)OC(=O)N1CC=C([Si](C)(C)Cc2ccccc2)CC1</smiles>

To a 250-mL round-bottomed flask, added $7.2 \mathrm{~g}$ (16.8 mmol) 4 and $170 \mathrm{~mL} \mathrm{CH} \mathrm{Cl}_{2}$. Placed, under a nitrogen atmosphere, in an ice bath. When the internal temperature fell below $4{ }^{\circ} \mathrm{C}$, added $2.8 \mathrm{~mL}(25.7 \mathrm{mmol})$ 1-chloroethylchloroformate. Let temperature rise gradually to $18{ }^{\circ} \mathrm{C}$ over about 2 hours, then heated to reflux via heating mantle. Let stir at reflux for 2 hours. Next, let cool to room temperature, removed solvent in vacuo, added $170 \mathrm{~mL} \mathrm{MeOH}$, and then let stir at reflux for 1 hour. After letting cool to room temperature, added $85 \mathrm{~mL}$ 1,4-dioxane, $85 \mathrm{~mL} 1 \mathrm{~N}$ aqueous $\mathrm{KOH}$ solution, and $5.5 \mathrm{~g}$ (25.2 mmol) di-t-butyl dicarbonate. Let stir at room temperature overnight. The following day, extracted 3 times with $250 \mathrm{~mL}$ methyl $t$-butyl ether, dried with $\mathrm{Na}_{2} \mathrm{SO}_{4}$, and purified by silica gel chromatography (automated column, $120 \mathrm{~g} \mathrm{SiO}_{2}, 0 \%$ to $10 \%$ EtOAc in hexanes gradient). Partitioned column fractions into 2 parts: first part contained $3.0 \mathrm{~g}$ of a yellow oil, which was shown to be 7 (ca. $90 \%$ purity by ${ }^{1} \mathrm{H} \mathrm{NMR}$ ); second part contained $1.8 \mathrm{~g}$ of a yellow oil that became a solid upon refrigeration, which was shown to be 7 (>95\% purity by ${ }^{1} \mathrm{H}$ NMR). Overall yield was approximately $80 \%$. ${ }^{1} \mathrm{H}$ NMR $\left(600 \mathrm{MHz}, \mathrm{DMSO}-\mathrm{d}_{6}, 80{ }^{\circ} \mathrm{C}\right): \delta=7.18(2 \mathrm{H}, \mathrm{t}, J=7.5 \mathrm{~Hz}), 7.04(1 \mathrm{H}, \mathrm{t}, J=7.3$ $\mathrm{Hz}), 6.99(2 \mathrm{H}, \mathrm{d}, J=7.6 \mathrm{~Hz}), 5.92(1 \mathrm{H}, \mathrm{s}), 3.83(2 \mathrm{H}, \mathrm{m}), 3.34(2 \mathrm{H}, \mathrm{t}, J=5.6 \mathrm{~Hz}), 2.15$ $(2 \mathrm{H}, \mathrm{s}), 2.05(2 \mathrm{H}, \mathrm{br}), 1.43(9 \mathrm{H}, \mathrm{s}), 0.030(6 \mathrm{H}, \mathrm{s}) .{ }^{13} \mathrm{C}$ NMR (150 MHz, DMSO-d 6,80 $\left.{ }^{\circ} \mathrm{C}\right): \delta=153.5,139.1,134.9,133.2,127.4,127.3,123.3,78.1,43.9,39.5,27.6,26.0$, 23.8, -5.0. HRMS: $\mathrm{M}+\mathrm{H}$ calculated for $\mathrm{C}_{19} \mathrm{H}_{29} \mathrm{NO}_{2} \mathrm{Si}=332.2040$; observed $\mathrm{m} / \mathrm{z}=$ 332.2036 (-1.3 ppm). Note: Applied the more pure portion of 7 to the cross-coupling reaction, generating known compound $\mathbf{8} .^{14 \mathrm{~b}}$

Representative procedure for the cross-coupling of aryl iodides. To a 4-mL vial, added $100 \mathrm{mg}(0.28 \mathrm{mmol}) 6$ and $6 \mathrm{mg}(0.0058 \mathrm{mmol})$ tris(dibenzylideneacetone)dipalladium(0)-chloroform adduct $\left(\mathrm{Pd}_{2} \mathrm{dba}_{3}-\mathrm{CHCl}_{3}\right)$. Next added $0.3 \mathrm{~mL} \mathrm{THF}, 48 \mu \mathrm{L}$ $(0.43 \mathrm{mmol})$ iodobenzene, and finally $1.1 \mathrm{~mL}(1.1 \mathrm{mmol}) \mathrm{TBAF}$ solution (1 M in THF). [Reaction quickly became clear upon TBAF addition.] Let stir at room temperature for 18 hours. At the end of the reaction, added $200 \mu \mathrm{L}$ triethylamine [in order to neutralize any residual acid], let stir briefly, concentrated in vacuo, and purified the crude material 
directly by silica gel chromatography (automated column, $12 \mathrm{~g} \mathrm{SiO}_{2}, 0 \%$ to $20 \%$ EtOAc in hexanes gradient). Obtained $63 \mathrm{mg}$ (90\%) of 1-benzyl-4-phenyl-1,2,3,6-tetrahydropyridine as a yellow oil.

Representative procedure for the cross-coupling of aryl bromides. To a 4-mL vial, added $100 \mathrm{mg}(0.28 \mathrm{mmol}) \mathbf{6}, 4 \mathrm{mg}(0.015 \mathrm{mmol})$ palladium(II) bromide $\left(\mathrm{PdBr}_{2}\right)$, and 8 $\mathrm{mg}(0.027 \mathrm{mmol}) 2$-(di- $t$-butylphosphino)biphenyl $\left(2-\left(t-\mathrm{Bu}_{2} \mathrm{P}\right) \mathrm{bp}\right)$. Next added $0.3 \mathrm{~mL}$ THF, $44 \mu \mathrm{L}(0.42 \mathrm{mmol})$ bromobenzene, and finally $1.1 \mathrm{~mL}(1.1 \mathrm{mmol})$ TBAF solution (1 $\mathrm{M}$ in THF). [Reaction quickly became clear upon TBAF addition.] Let stir at $50{ }^{\circ} \mathrm{C}$ for 20 hours. At the end of the reaction, added $200 \mu \mathrm{L}$ triethylamine [in order to neutralize any residual acid], let stir briefly, concentrated in vacuo, and purified the crude material directly by silica gel chromatography (automated column, $12 \mathrm{~g} \mathrm{SiO}_{2}, 0 \%$ to $20 \%$ EtOAc in hexanes gradient). Obtained $52 \mathrm{mg}$ (74\%) of 1-benzyl-4-phenyl-1,2,3,6tetrahydro-pyridine as a yellow oil.

\section{1-Benzyl-4-phenyl-1,2,3,6-tetrahydro-pyridine}<smiles>C1=C(c2ccccc2)CCN(Cc2ccccc2)C1</smiles>

${ }^{1} \mathrm{H}$ NMR $\left(400 \mathrm{MHz}, \mathrm{CDCl}_{3}\right): \delta=7.30(10 \mathrm{H}, \mathrm{m}), 6.05(1 \mathrm{H}, \mathrm{m}), 3.63(2 \mathrm{H}, \mathrm{s}), 3.17(2 \mathrm{H}$, $\mathrm{m}), 2.71(2 \mathrm{H}, \mathrm{t}, J=5.7 \mathrm{~Hz}), 2.56(2 \mathrm{H}, \mathrm{m}) .{ }^{13} \mathrm{C} \mathrm{NMR}\left(125 \mathrm{MHz}, \mathrm{CDCl}_{3}\right): \delta=140.9$, 138.2, 134.9, 129.2, 128.23, 128.21, 127.1, 126.9, 124.9, 121.9, 62.7, 53.3, 49.9, 28.0.

HRMS: $\mathrm{M}+\mathrm{H}$ calculated for $\mathrm{C}_{18} \mathrm{H}_{19} \mathrm{~N}=250.1590$; observed $\mathrm{m} / \mathrm{z}=250.1602$ (-4.7 ppm).

\section{1-Benzyl-4-p-tolyl-1,2,3,6-tetrahydro-pyridine}<smiles>Cc1ccc(C2=CCN(Cc3ccccc3)CC2)cc1</smiles>

${ }^{1} \mathrm{H}$ NMR $\left(500 \mathrm{MHz}, \mathrm{CDCl}_{3}\right): \delta=7.33(7 \mathrm{H}, \mathrm{m}), 7.11(2 \mathrm{H}, \mathrm{d}, J=7.9 \mathrm{~Hz}), 6.02(1 \mathrm{H}, \mathrm{m})$, $3.63(2 \mathrm{H}, \mathrm{s}), 3.16(2 \mathrm{H}, \mathrm{m}), 2.70(2 \mathrm{H}, \mathrm{t}, J=5.7 \mathrm{~Hz}), 2.54(2 \mathrm{H}, \mathrm{m}), 2.32(3 \mathrm{H}, \mathrm{s}) .{ }^{13} \mathrm{C} \mathrm{NMR}$ $\left(125 \mathrm{MHz}, \mathrm{CDCl}_{3}\right): \delta=138.3,138.0,136.5,134.7,129.2,128.9,128.2,127.0,124.7$, 121.0, 62.7, 53.3, 50.0, 28.0, 21.0. HRMS: $\mathrm{M}+\mathrm{H}$ calculated for $\mathrm{C}_{19} \mathrm{H}_{21} \mathrm{~N}=264.1747$; observed $\mathrm{m} / \mathrm{z}=264.1741(2.2 \mathrm{ppm})$. 


\section{1-Benzyl-4-o-tolyl-1,2,3,6-tetrahydro-pyridine}<smiles>Cc1ccccc1C1=CCN(Cc2ccccc2)CC1</smiles>

${ }^{1} \mathrm{H}$ NMR $\left(500 \mathrm{MHz}, \mathrm{CDCl}_{3}\right): \delta=7.37(2 \mathrm{H}, \mathrm{d}, J=8.3 \mathrm{~Hz}), 7.32(2 \mathrm{H}, \mathrm{t}, J=7.4 \mathrm{~Hz}), 7.25$ $(1 \mathrm{H}, \mathrm{t}, J=7.2 \mathrm{~Hz}), 7.11(4 \mathrm{H}, \mathrm{m}), 5.51(1 \mathrm{H}, \mathrm{m}), 3.64(2 \mathrm{H}, \mathrm{s}), 3.13(2 \mathrm{H}, \mathrm{m}), 2.67(2 \mathrm{H}, \mathrm{t}, J$ $=5.6 \mathrm{~Hz}), 2.35(2 \mathrm{H}, \mathrm{m}), 2.28(3 \mathrm{H}, \mathrm{s}) .{ }^{13} \mathrm{C} \mathrm{NMR}\left(125 \mathrm{MHz} \mathrm{CDCl}_{3}\right): \delta=142.9,138.2$, 137.0, 135.0, 130.0, 129.2, 128.2, 127.0, 126.7, 125.5, 123.6, 62.7, 52.9, 49.8, 30.7, 19.9 . HRMS: $\mathrm{M}+\mathrm{H}$ calculated for $\mathrm{C}_{19} \mathrm{H}_{21} \mathrm{~N}=264.1747$; observed $\mathrm{m} / \mathrm{z}=264.1746$ (0.3 ppm).

\section{1-Benzyl-4-(4-methoxy-phenyl)-1,2,3,6-tetrahydro-pyridine}<smiles>COc1ccc(C2=CCN(Cc3ccccc3)CC2)cc1</smiles>

${ }^{1} \mathrm{H}$ NMR $\left(500 \mathrm{MHz}, \mathrm{CDCl}_{3}\right): \delta=7.30(7 \mathrm{H}, \mathrm{m}), 6.83(2 \mathrm{H}, \mathrm{d}, J=8.9 \mathrm{~Hz}), 5.96(1 \mathrm{H}, \mathrm{m})$, $3.78(3 \mathrm{H}, \mathrm{s}), 3.62(2 \mathrm{H}, \mathrm{s}), 3.15(2 \mathrm{H}, \mathrm{m}), 2.69(2 \mathrm{H}, \mathrm{t}, J=5.7 \mathrm{~Hz}), 2.52(2 \mathrm{H}, \mathrm{m}) .{ }^{13} \mathrm{C} \mathrm{NMR}$ $\left(125 \mathrm{MHz}, \mathrm{CDCl}_{3}\right): \delta=158.6,138.3,134.2,133.5,129.2,128.2,127.0,125.9,120.2$, 113.6, 62.7, 55.2, 53.3, 50.0, 28.1. HRMS: $\mathrm{M}+\mathrm{H}$ calculated for $\mathrm{C}_{19} \mathrm{H}_{21} \mathrm{NO}=280.1696$; observed $\mathrm{m} / \mathrm{z}=280.1702(-2.2 \mathrm{ppm})$.

\section{1-Benzyl-4-(2-methoxy-phenyl)-1,2,3,6-tetrahydro-pyridine}<smiles>COc1ccccc1C1=CCN(Cc2ccccc2)CC1</smiles>

${ }^{1} \mathrm{H}$ NMR $\left(500 \mathrm{MHz}, \mathrm{CDCl}_{3}\right): \delta=7.40(2 \mathrm{H}, \mathrm{d}, J=7.1 \mathrm{~Hz}), 7.34(2 \mathrm{H}, \mathrm{t}, J=7.5 \mathrm{~Hz}), 7.22$ $(3 \mathrm{H}, \mathrm{m}), 6.91(1 \mathrm{H}, \mathrm{td}, J=7.4,0.77 \mathrm{~Hz}), 6.86(1 \mathrm{H}, \mathrm{d}, J=8.2 \mathrm{~Hz}), 5.79(1 \mathrm{H}, \mathrm{m}), 3.80$ $(3 \mathrm{H}, \mathrm{s}), 3.66(2 \mathrm{H}, \mathrm{s}), 3.18(2 \mathrm{H}, \mathrm{m}), 2.69(2 \mathrm{H}, \mathrm{t}, J=5.7 \mathrm{~Hz}), 2.56(2 \mathrm{H}, \mathrm{m}) .{ }^{13} \mathrm{C} \mathrm{NMR}(125$ $\left.\mathrm{MHz}_{\mathrm{CDCl}}\right): \delta=156.8,138.2,135.5,131.9,129.4,129.2,128.2,128.0,127.0,123.9$, 120.5, 110.7, 62.8, 55.3, 53.2, 49.9, 29.5. HRMS: $\mathrm{M}+\mathrm{H}$ calculated for $\mathrm{C}_{19} \mathrm{H}_{21} \mathrm{NO}=$ 280.1696; observed $\mathrm{m} / \mathrm{z}=280.1697$ (-0.4 ppm). 


\section{1-Benzyl-4-thiophen-2-yl-1,2,3,6-tetrahydro-pyridine}<smiles>C1=C(c2cccs2)CCN(Cc2ccccc2)C1</smiles>

${ }^{1} \mathrm{H}$ NMR (400 MHz, $\left.\mathrm{CDCl}_{3}\right): \delta=7.32(5 \mathrm{H}, \mathrm{m}), 7.10(1 \mathrm{H}, \mathrm{dd}, J=4.6,1.6 \mathrm{~Hz}), 6.94(2 \mathrm{H}$, $\mathrm{m}), 6.07(1 \mathrm{H}, \mathrm{m}), 3.62(2 \mathrm{H}, \mathrm{s}), 3.14(2 \mathrm{H}, \mathrm{m}), 2.69(2 \mathrm{H}, \mathrm{t}, J=5.7 \mathrm{~Hz}), 2.56(2 \mathrm{H}, \mathrm{m}) .{ }^{13} \mathrm{C}$ $\operatorname{NMR}\left(125 \mathrm{MHz}, \mathrm{CDCl}_{3}\right): \delta=145.4,138.2,129.6,129.1,128.2,127.12,127.08,123.1$, 121.7, 121.0, 62.5, 52.9, 49.6, 28.3. HRMS: $\mathrm{M}+\mathrm{H}$ calculated for $\mathrm{C}_{16} \mathrm{H}_{17} \mathrm{NS}=256.1154$; observed $\mathrm{m} / \mathrm{z}=256.1160(-2.2 \mathrm{ppm})$.

\section{1-Benzyl-4-thiophen-3-yl-1,2,3,6-tetrahydro-pyridine}<smiles>C1=C(c2ccsc2)CCN(Cc2ccccc2)C1</smiles>

${ }^{1} \mathrm{H}$ NMR $\left(500 \mathrm{MHz}, \mathrm{CDCl}_{3}\right): \delta=7.28(7 \mathrm{H}, \mathrm{m}), 7.06(1 \mathrm{H}, \mathrm{m}), 6.04(1 \mathrm{H}, \mathrm{m}), 3.61(2 \mathrm{H}, \mathrm{s})$, $3.13(2 \mathrm{H}, \mathrm{m}), 2.68(2 \mathrm{H}, \mathrm{t}, J=5.7 \mathrm{~Hz}), 2.52(2 \mathrm{H}, \mathrm{m}) .{ }^{13} \mathrm{C} \mathrm{NMR}\left(125 \mathrm{MHz}, \mathrm{CDCl}_{3}\right): \delta=$ 142.6, 138.3, 130.4, 129.1, 128.2, 127.0, 125.3, 124.6, 121.0, 118.6, 62.6, 53.0, 49.7, 28.0. HRMS: $\mathrm{M}+\mathrm{H}$ calculated for $\mathrm{C}_{16} \mathrm{H}_{17} \mathrm{NS}=256.1154$; observed $\mathrm{m} / \mathrm{z}=256.1163(-3.3$ ppm).

\section{1-Benzyl-4-(4-fluoro-phenyl)-1,2,3,6-tetrahydro-pyridine}<smiles>Fc1ccc(C2=CCN(Cc3ccccc3)CC2)cc1</smiles>

${ }^{1} \mathrm{H}$ NMR $\left(400 \mathrm{MHz}, \mathrm{CDCl}_{3}\right): \delta=7.32(7 \mathrm{H}, \mathrm{m}), 6.98(2 \mathrm{H}, \mathrm{t}, J=8.8 \mathrm{~Hz}), 5.99(1 \mathrm{H}, \mathrm{m})$, $3.63(2 \mathrm{H}, \mathrm{s}), 3.15(2 \mathrm{H}, \mathrm{m}), 2.70(2 \mathrm{H}, \mathrm{t}, J=5.7 \mathrm{~Hz}), 2.52(2 \mathrm{H}, \mathrm{m}) .{ }^{13} \mathrm{C}$ NMR $(125 \mathrm{MHz}$, $\left.\mathrm{CDCl}_{3}\right): \delta=162.0(\mathrm{~d}, J=246 \mathrm{~Hz}), 138.2,137.0(\mathrm{~d}, J=3.2 \mathrm{~Hz}), 134.0,129.2,128.3$, 127.1, $126.4(\mathrm{~d}, J=7.8 \mathrm{~Hz}), 121.8,115.0(\mathrm{~J}=21 \mathrm{~Hz}), 62.7,53.2$, 49.9, 28.2. HRMS: $\mathrm{M}+\mathrm{H}$ calculated for $\mathrm{C}_{18} \mathrm{H}_{18} \mathrm{NF}=268.1496$; observed $\mathrm{m} / \mathrm{z}=268.1506(-3.7 \mathrm{ppm})$. 


\section{1-Benzyl-4-(4-trifluoromethyl-phenyl)-1,2,3,6-tetrahydro-pyridine}<smiles>FC(F)(F)c1ccc(C2=CCN(Cc3ccccc3)CC2)cc1</smiles>

${ }^{1} \mathrm{H}$ NMR $\left(400 \mathrm{MHz}, \mathrm{CDCl}_{3}\right): \delta=7.50(2 \mathrm{H}, \mathrm{d}, J=8.3 \mathrm{~Hz}), 7.40(2 \mathrm{H}, \mathrm{d}, J=8.2 \mathrm{~Hz}), 7.29$ $(5 \mathrm{H}, \mathrm{m}), 6.09(1 \mathrm{H}, \mathrm{m}), 3.59(2 \mathrm{H}, \mathrm{s}), 3.13(2 \mathrm{H}, \mathrm{m}), 2.66(2 \mathrm{H}, \mathrm{t}, J=5.7 \mathrm{~Hz}), 2.49(2 \mathrm{H}, \mathrm{m})$. ${ }^{13} \mathrm{C} \mathrm{NMR}\left(100 \mathrm{MHz}, \mathrm{CDCl}_{3}\right): \delta=144.2,138.0,133.8,129.1,128.7$ (q, $\left.J=32 \mathrm{~Hz}\right)$, 128.2, 127.1, 125.1 (q, $J=3.8 \mathrm{~Hz}), 124.9,124.3(\mathrm{q}, J=272 \mathrm{~Hz}), 124.1,62.6,53.1,49.6$, 27.8. HRMS: $\mathrm{M}+\mathrm{H}$ calculated for $\mathrm{C}_{19} \mathrm{H}_{18} \mathrm{NF}_{3}=318.1464$; observed $\mathrm{m} / \mathrm{z}=318.1468(-$ $1.2 \mathrm{ppm})$.

\section{1-[4-(1-Benzyl-1,2,3,6-tetrahydro-pyridin-4-yl)-phenyl]-ethanone}<smiles>CC(=O)c1ccc(C2=CCN(Cc3ccccc3)CC2)cc1</smiles>

${ }^{1} \mathrm{H}$ NMR $\left(400 \mathrm{MHz}, \mathrm{CDCl}_{3}\right): \delta=7.88(2 \mathrm{H}, \mathrm{d}, J=8.6 \mathrm{~Hz}), 7.44(2 \mathrm{H}, \mathrm{d}, J=8.5 \mathrm{~Hz}), 7.30$ $(5 \mathrm{H}, \mathrm{m}), 6.19(1 \mathrm{H}, \mathrm{m}), 3.62(2 \mathrm{H}, \mathrm{s}), 3.17(2 \mathrm{H}, \mathrm{m}), 2.71(2 \mathrm{H}, \mathrm{t}, J=5.7 \mathrm{~Hz}), 2.56(2 \mathrm{H}, \mathrm{m})$, 2.55 (3H. s). ${ }^{13} \mathrm{C} \mathrm{NMR}\left(125 \mathrm{MHz}, \mathrm{CDCl}_{3}\right): \delta=197.5,145.3,138.0,135.5,134.1,129.1$, 128.4, 128.2, 127.1, 124.8, 124.5, 62.6, 53.2, 49.7, 27.8, 26.4. HRMS: M+H calculated for $\mathrm{C}_{20} \mathrm{H}_{21} \mathrm{NO}=292.1696$; observed $\mathrm{m} / \mathrm{z}=292.1707$ (-3.8 ppm).

\section{4-(1-Benzyl-1,2,3,6-tetrahydro-pyridin-4-yl)-benzoic acid methyl ester}<smiles>COC(=O)c1ccc(C2=CCN(Cc3ccccc3)CC2)cc1</smiles>

${ }^{1} \mathrm{H}$ NMR $\left(500 \mathrm{MHz}, \mathrm{CDCl}_{3}\right): \delta=7.97(2 \mathrm{H}, \mathrm{d}, J=8.5 \mathrm{~Hz}), 7.43(2 \mathrm{H}, \mathrm{d}, J=8.5 \mathrm{~Hz}), 7.34$ $(5 \mathrm{H}, \mathrm{m}), 6.19(1 \mathrm{H}, \mathrm{m}), 3.89(3 \mathrm{H}, \mathrm{s}), 3.63(2 \mathrm{H}, \mathrm{s}), 3.18(2 \mathrm{H}, \mathrm{m}), 2.71(2 \mathrm{H}, \mathrm{t}, J=5.7 \mathrm{~Hz})$, $2.56(2 \mathrm{H}, \mathrm{m}) .{ }^{13} \mathrm{C}$ NMR $\left(125 \mathrm{MHz}, \mathrm{CDCl}_{3}\right): \delta=166.9,145.1,138.1,134.2,129.6$, 129.1, 128.4, 128.2, 127.1, 124.6, 124.2, 62.6, 53.3, 51.9, 49.7, 27.8. HRMS: M+H calculated for $\mathrm{C}_{20} \mathrm{H}_{21} \mathrm{NO}_{2}=308.1645$; observed $\mathrm{m} / \mathrm{z}=308.1647$ (-0.6 ppm). 


\section{4-(1-Benzyl-1,2,3,6-tetrahydro-pyridin-4-yl)-benzonitrile}<smiles>N#Cc1ccc(C2=CCN(Cc3ccccc3)CC2)cc1</smiles>

${ }^{1} \mathrm{H}$ NMR $\left(500 \mathrm{MHz}, \mathrm{CDCl}_{3}\right): \delta=7.58(2 \mathrm{H}, \mathrm{d}, J=8.6 \mathrm{~Hz}), 7.45(2 \mathrm{H}, \mathrm{d}, J=8.5 \mathrm{~Hz}), 7.32$ $(5 \mathrm{H}, \mathrm{m}), 6.20(1 \mathrm{H}, \mathrm{m}), 3.64(2 \mathrm{H}, \mathrm{s}), 3.19(2 \mathrm{H}, \mathrm{m}), 2.72(2 \mathrm{H}, \mathrm{t}, J=5.7 \mathrm{~Hz}), 2.53(2 \mathrm{H}, \mathrm{m})$. ${ }^{13} \mathrm{C} \mathrm{NMR}\left(125 \mathrm{MHz}, \mathrm{CDCl}_{3}\right): \delta=145.1,137.9,133.6,132.1,129.1,128.2,127.1,125.4$, 125.3, 119.0, 110.2, 62.5, 53.2, 49.6, 27.7. HRMS: $\mathrm{M}+\mathrm{H}$ calculated for $\mathrm{C}_{19} \mathrm{H}_{18} \mathrm{~N}_{2}=$ 275.1543; observed $\mathrm{m} / \mathrm{z}=275.1554(-4.1 \mathrm{ppm})$.

\section{1-Benzyl-4-(4-bromo-phenyl)-1,2,3,6-tetrahydro-pyridine}<smiles>Brc1ccc(C2=CCN(Cc3ccccc3)CC2)cc1</smiles>

${ }^{1} \mathrm{H}$ NMR $\left(400 \mathrm{MHz}, \mathrm{CDCl}_{3}\right): \delta=7.32(9 \mathrm{H}, \mathrm{m}), 6.05(1 \mathrm{H}, \mathrm{m}), 3.63(2 \mathrm{H}, \mathrm{s}), 3.14(2 \mathrm{H}, \mathrm{m})$, $2.70(2 \mathrm{H}, \mathrm{t}, J=5.7 \mathrm{~Hz}), 2.50(2 \mathrm{H}, \mathrm{m}) .{ }^{13} \mathrm{C} \mathrm{NMR}\left(125 \mathrm{MHz}, \mathrm{CDCl}_{3}\right): \delta=139.7,138.1$, 134.0, 131.3, 129.2, 128.3, 127.1, 126.5, 122.6, 120.7, 62.7, 53.2, 49.8, 27.9. HRMS: $\mathrm{M}+\mathrm{H}$ calculated for $\mathrm{C}_{18} \mathrm{H}_{18} \mathrm{NBr}=328.0695$; observed $\mathrm{m} / \mathrm{z}=328.0702(-2.0 \mathrm{ppm})$. 
$N$-(1-Benzyl-piperidin-4-ylidene)- $N$ '-tosyl-hydrazine (2)<smiles>Cc1ccc(S(=O)(=O)NN=C2CCN(Cc3ccccc3)CC2)cc1</smiles>
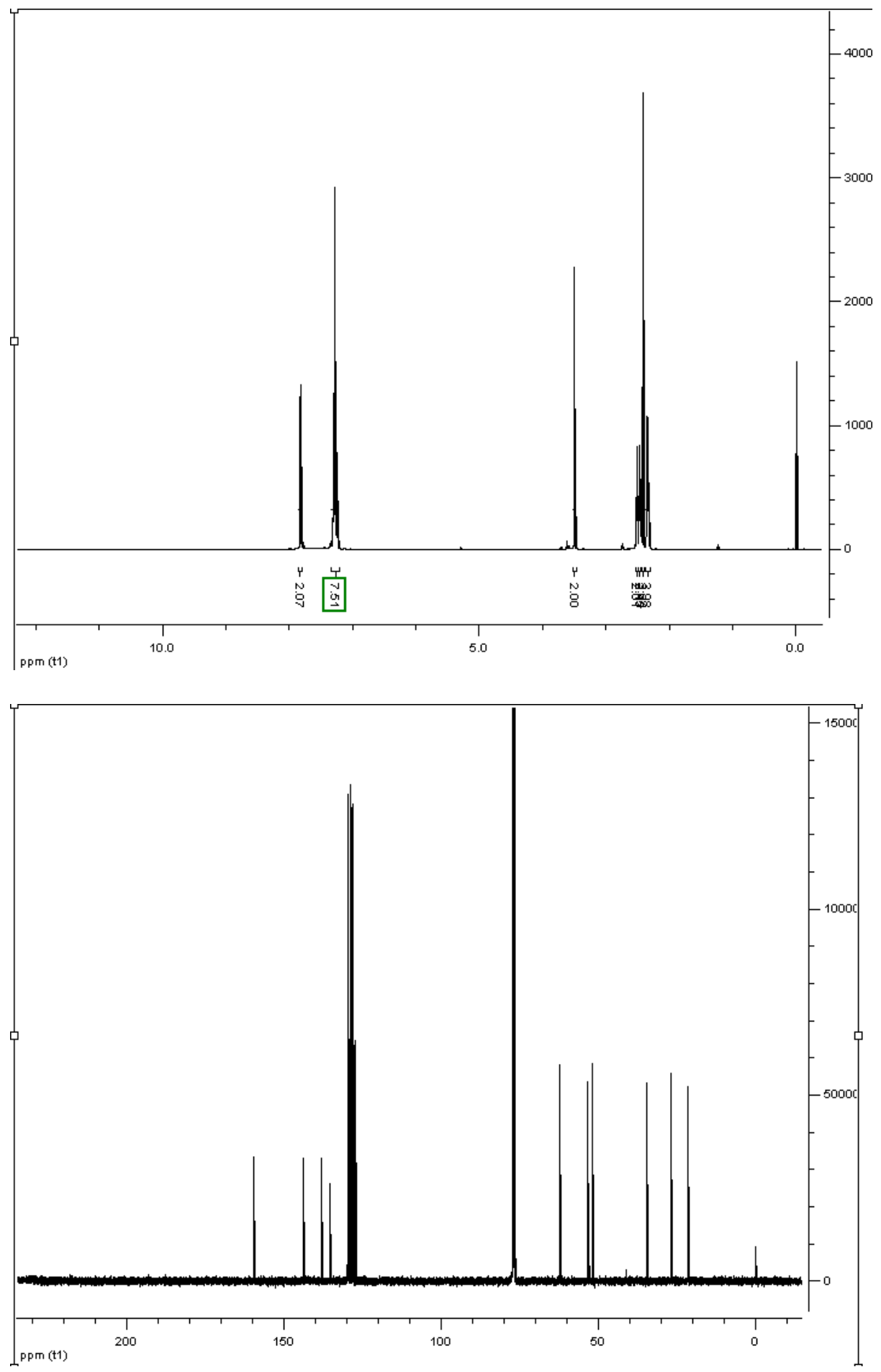
1-Benzyl-4-(benzyldimethylsilanyl)-1,2,3,6-tetrahydro-pyridine (4)<smiles>C[Si](C)(Cc1ccccc1)C1=CCN(Cc2ccccc2)CC1</smiles>
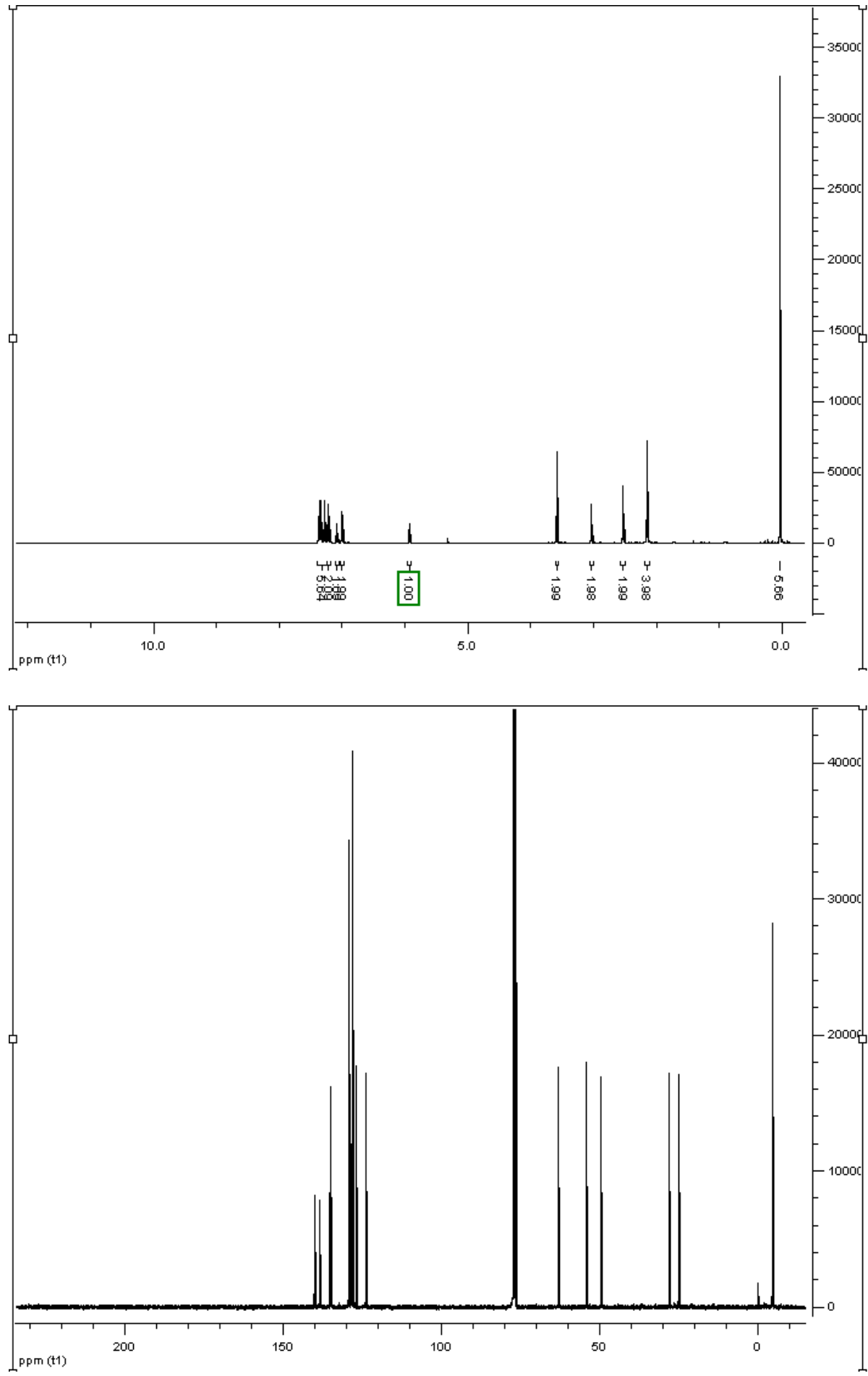
1-Benzyl-4-(benzyldimethylsilanyl)-1,2,3,6-tetrahydro-pyridine hydrochloride (6)<smiles>C[Si](Cc1ccccc1)(c1ccccc1)c1cc[nH][c+](CCl)c1</smiles>
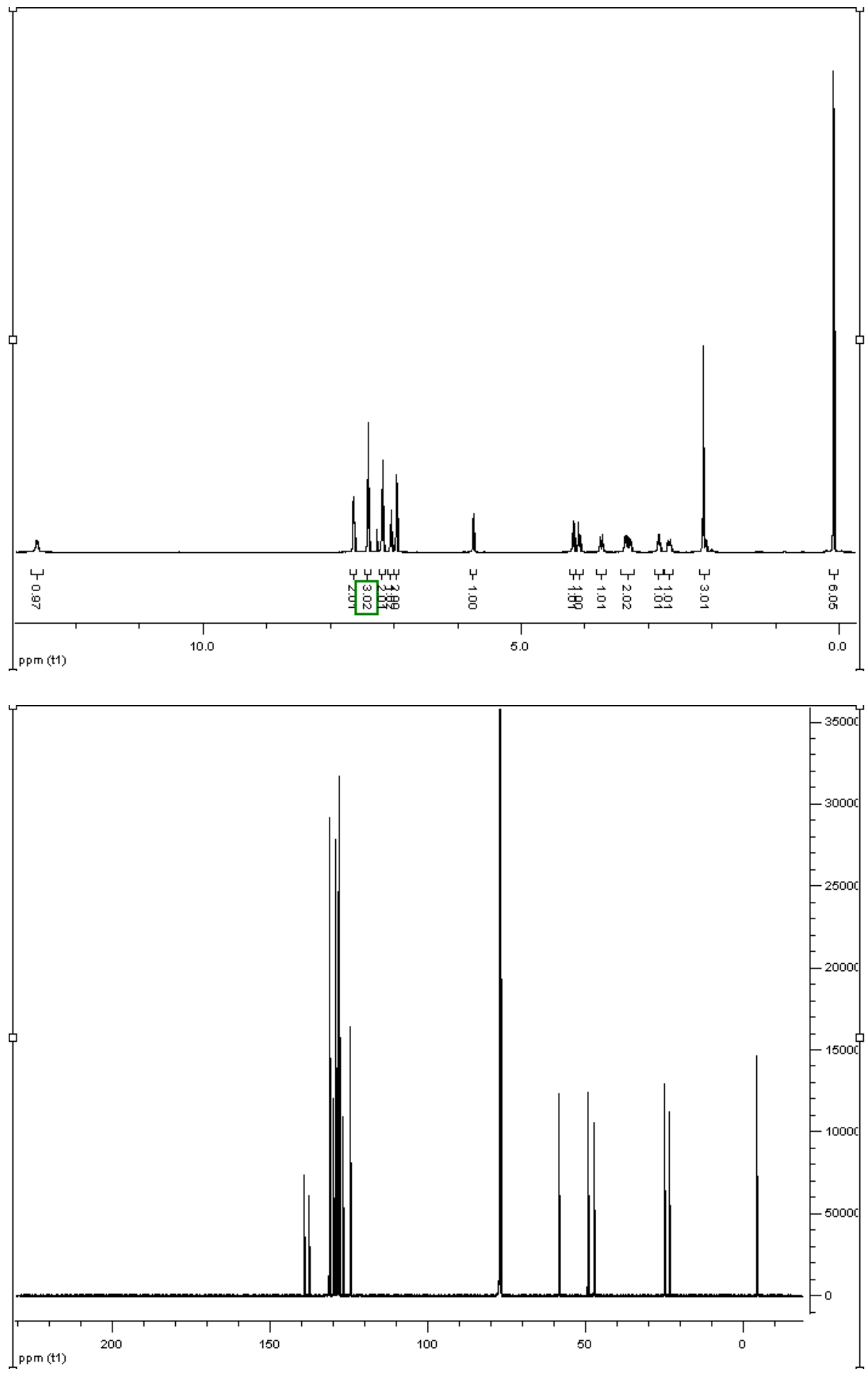
4-(Benzyldimethylsilanyl)-3,6-dihydro-2H-pyridine-1-t-butoxycarbonyl (7)<smiles>CC(C)(C)OC(=O)N1CC=C([Si](C)(C)Cc2ccccc2)CC1</smiles>
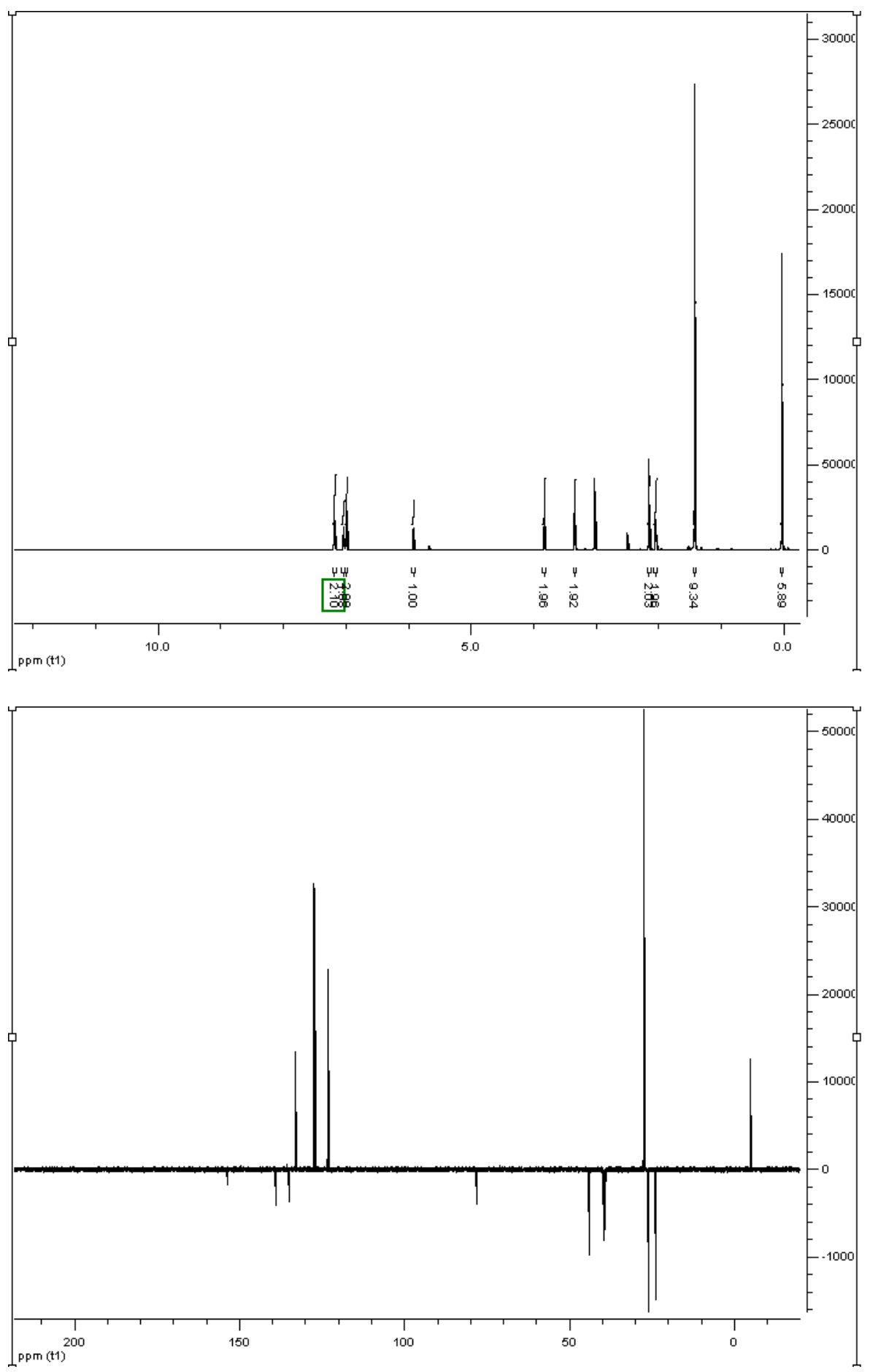
1-Benzyl-4-phenyl-1,2,3,6-tetrahydro-pyridine (Table 2, entry 1)<smiles>C1=C(c2ccccc2)CCN(Cc2ccccc2)C1</smiles>
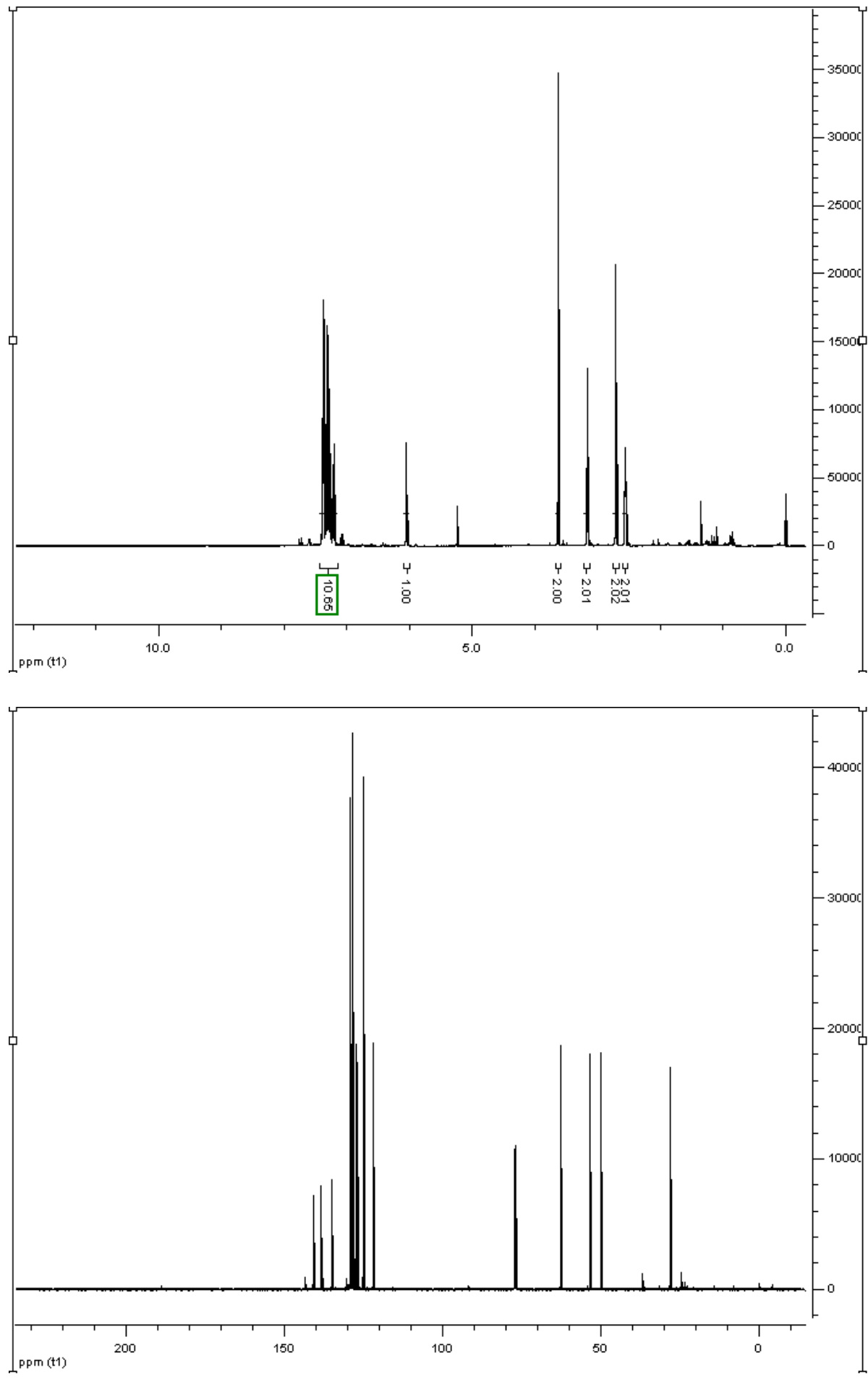
1-Benzyl-4-p-tolyl-1,2,3,6-tetrahydro-pyridine (Table 2, entry 2)<smiles>Cc1ccc(C2=CCN(Cc3ccccc3)CC2)cc1</smiles>
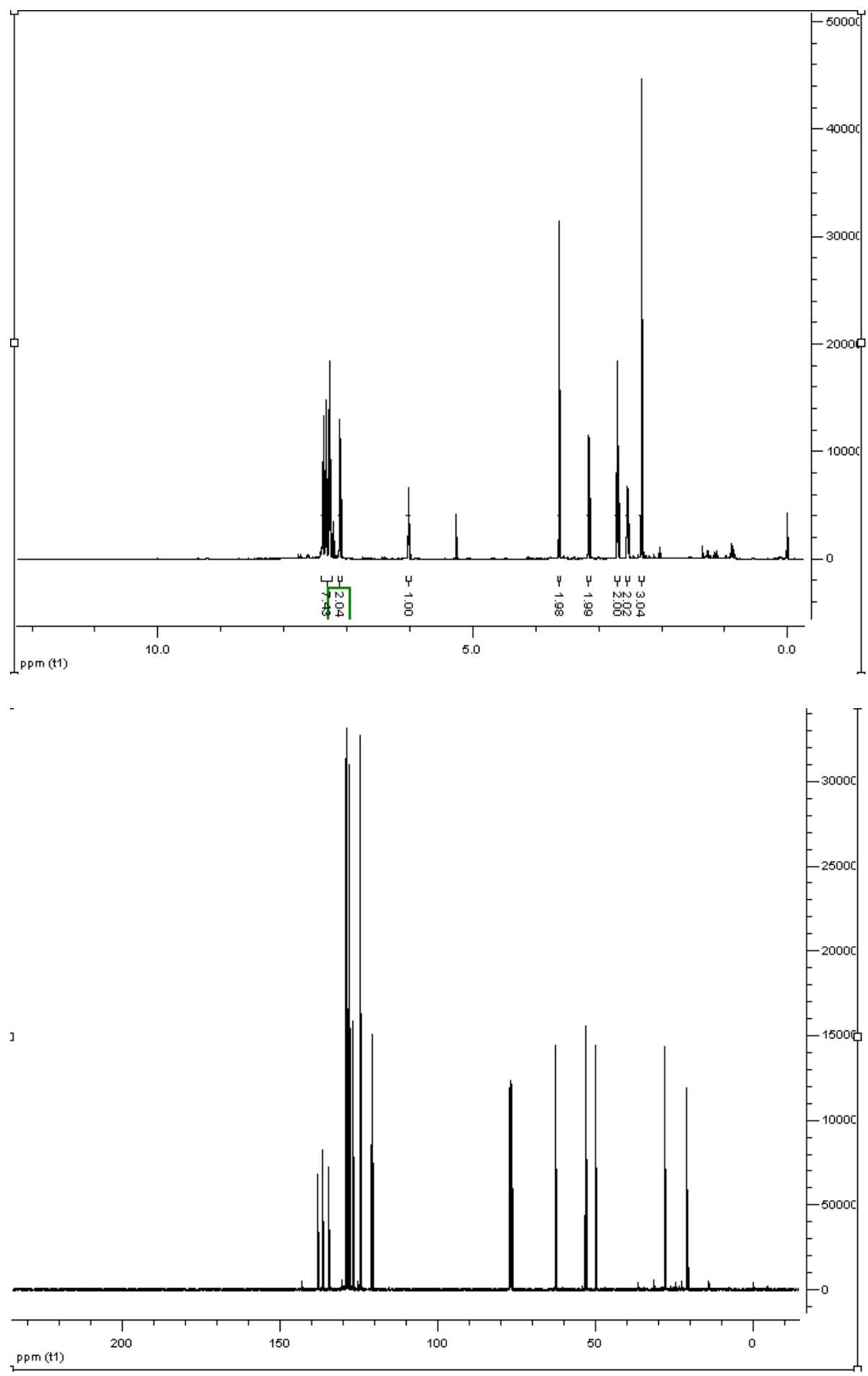
1-Benzyl-4-o-tolyl-1,2,3,6-tetrahydro-pyridine (Table 2, entry 4)<smiles>Cc1ccccc1C1=CCN(Cc2ccccc2)CC1</smiles>
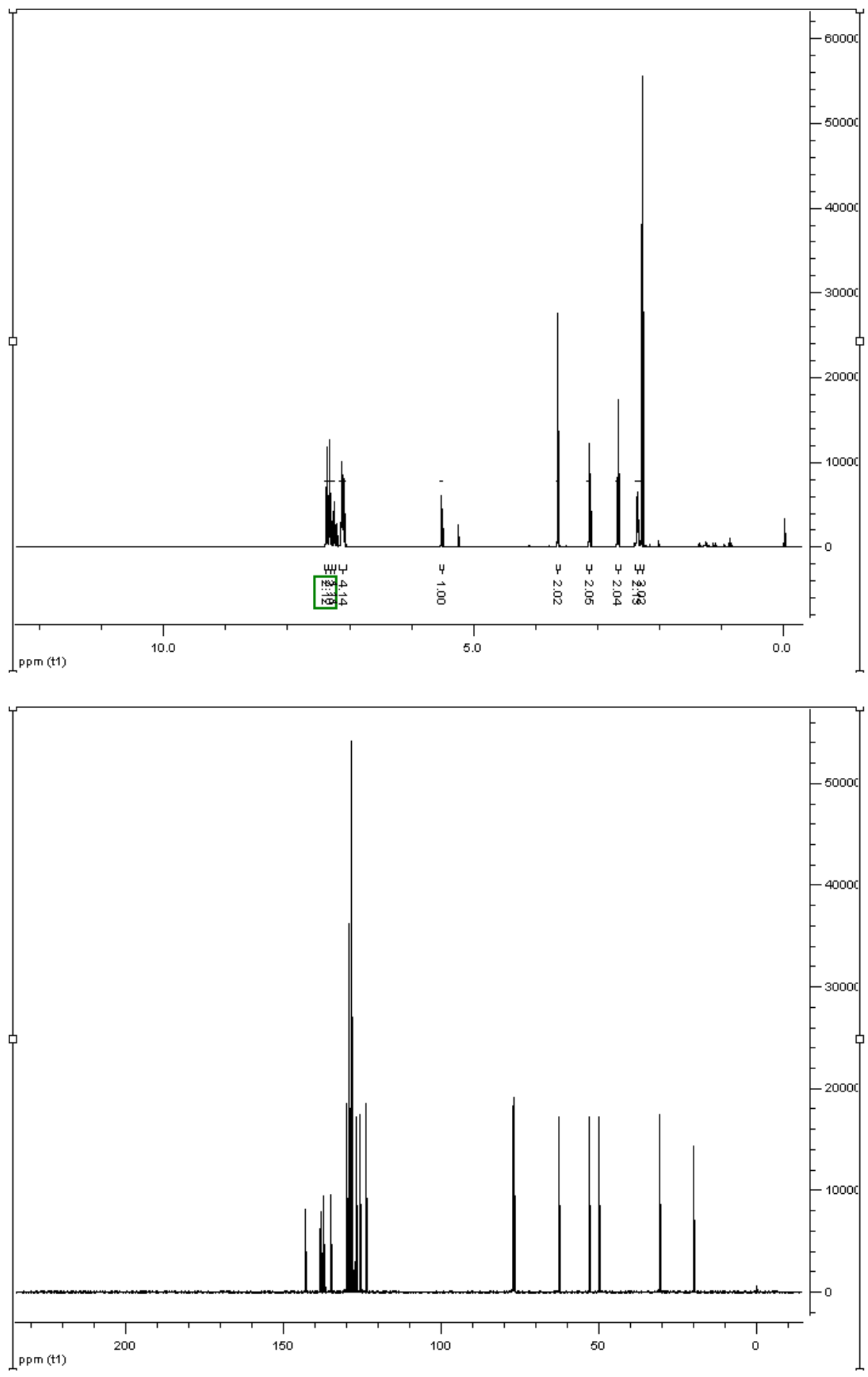
1-Benzyl-4-(4-methoxy-phenyl)-1,2,3,6-tetrahydro-pyridine (Table 2, entry 5)<smiles>COc1ccc(C2=CCN(Cc3ccccc3)CC2)cc1</smiles>
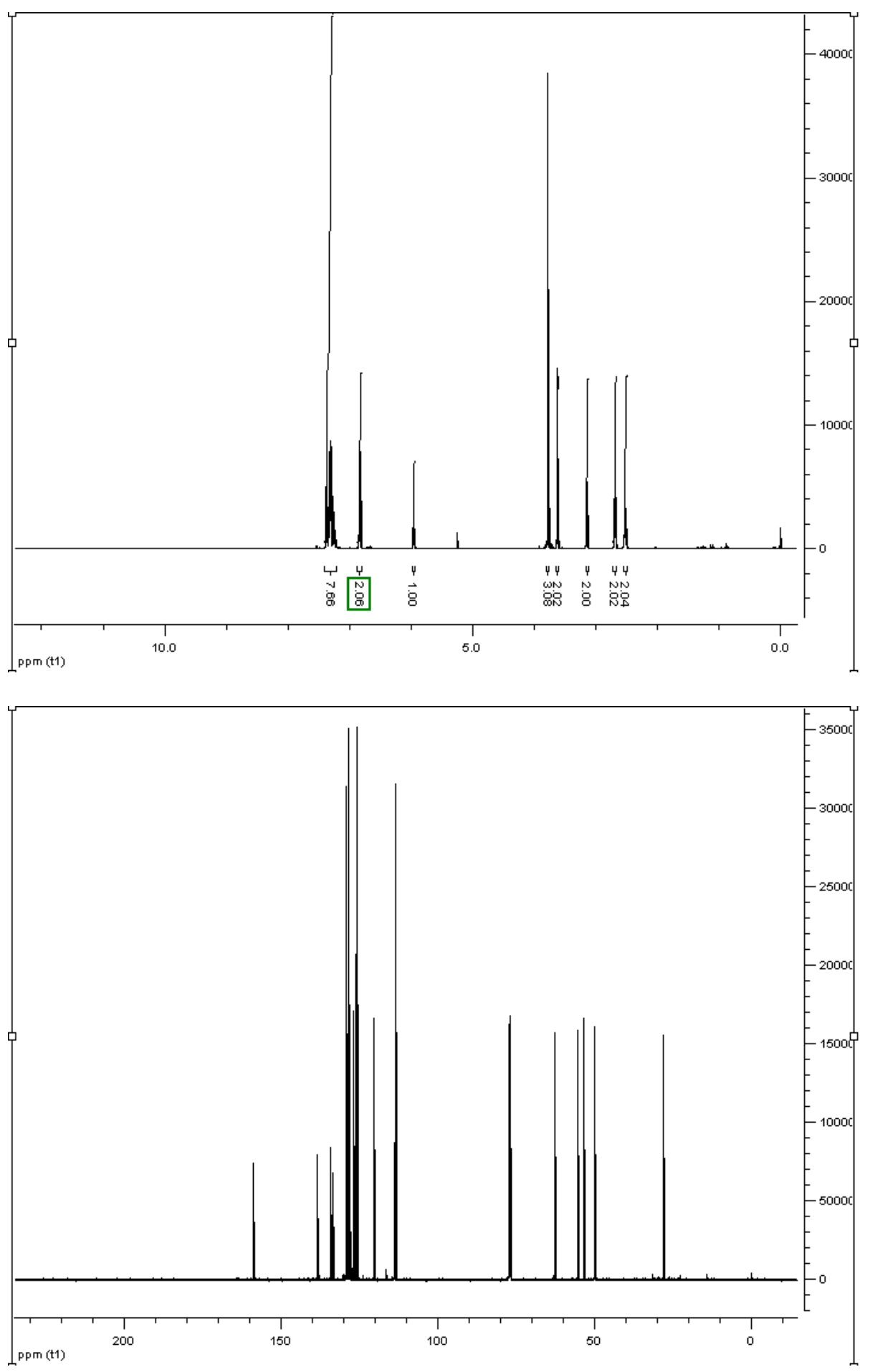
1-Benzyl-4-(2-methoxy-phenyl)-1,2,3,6-tetrahydro-pyridine (Table 2, entry 7)<smiles>COc1ccccc1C1=CCN(Cc2ccccc2)CC1</smiles>
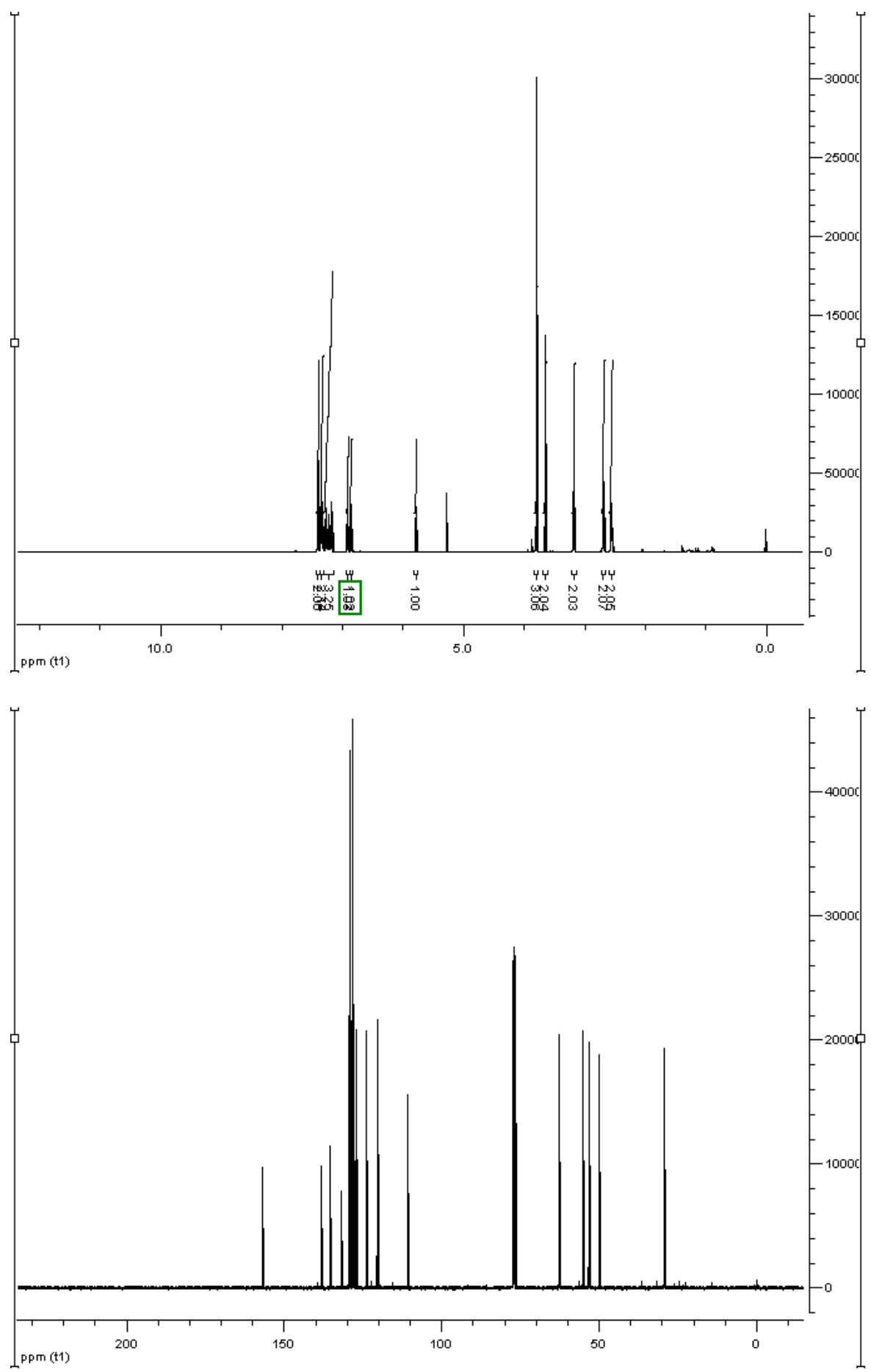
1-Benzyl-4-thiophen-2-yl-1,2,3,6-tetrahydro-pyridine (Table 2, entry 9)<smiles>C1=C(c2cccs2)CCN(Cc2ccccc2)C1</smiles>
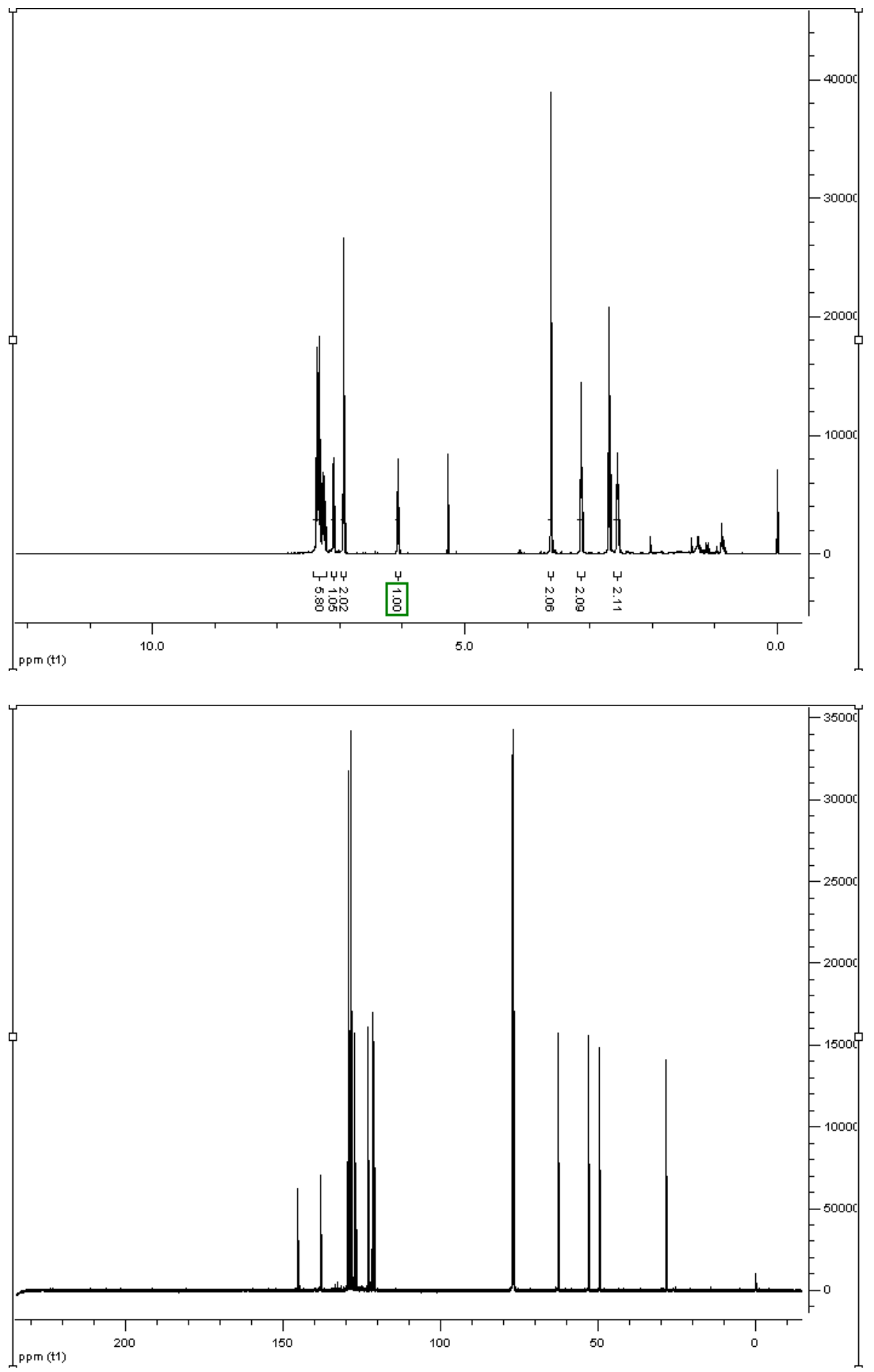
1-Benzyl-4-thiophen-3-yl-1,2,3,6-tetrahydro-pyridine (Table 2, entry 10)<smiles>C1=C(c2ccsc2)CCN(Cc2ccccc2)C1</smiles>
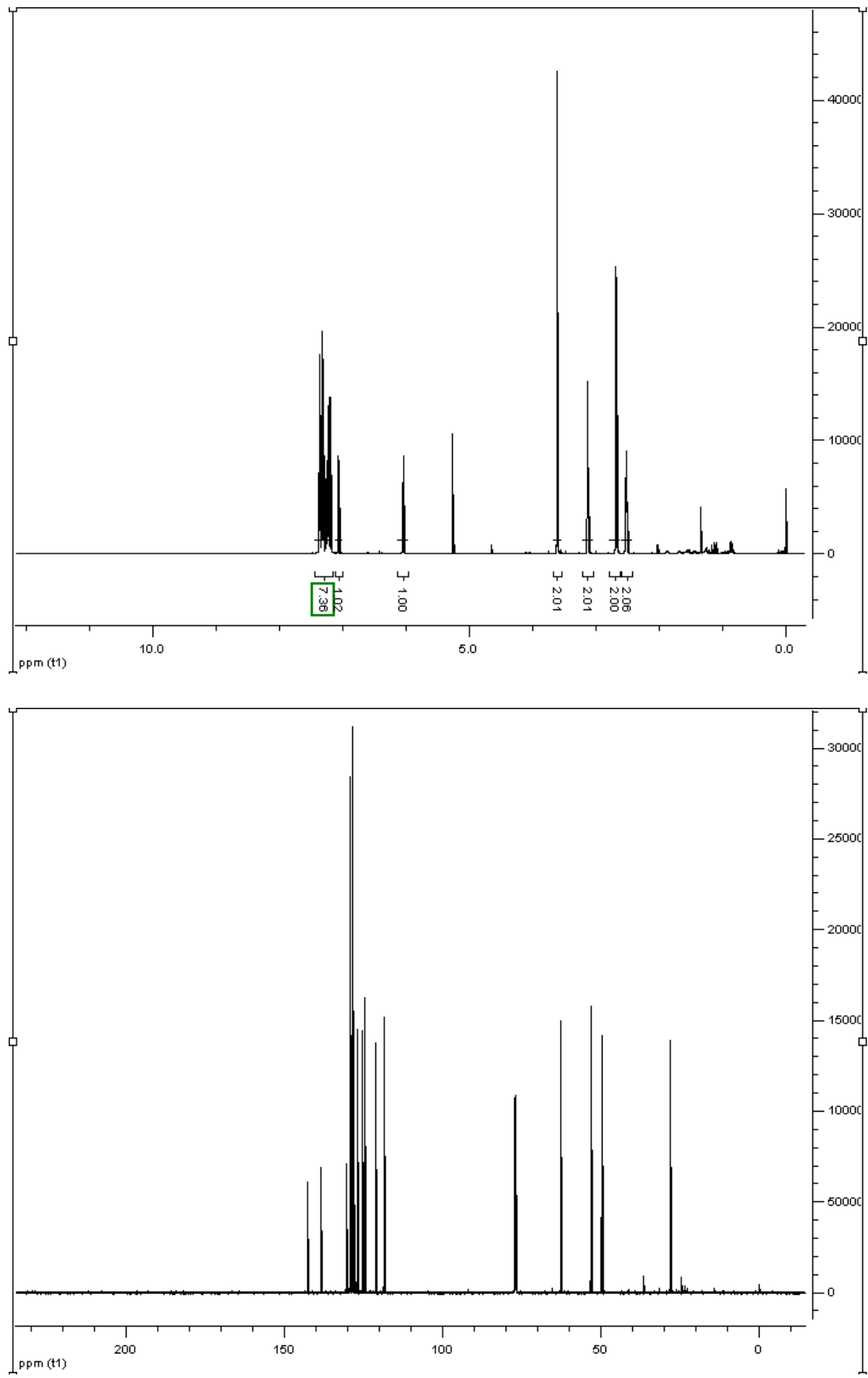
1-Benzyl-4-(4-fluoro-phenyl)-1,2,3,6-tetrahydro-pyridine (Table 2, entry 11)<smiles>Fc1ccc(C2=CCN(Cc3ccccc3)CC2)cc1</smiles>
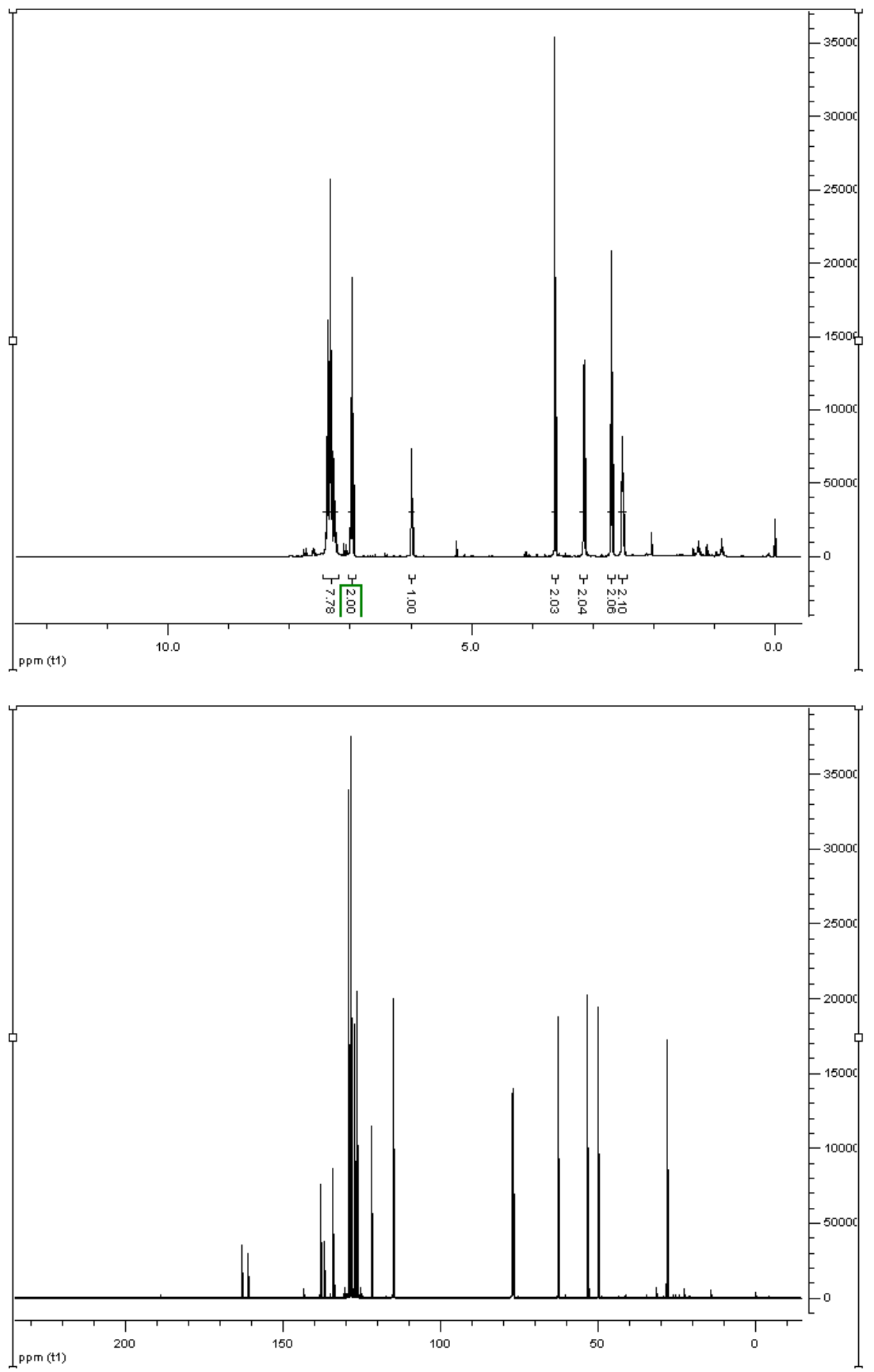
1-Benzyl-4-(4-trifluoromethyl-phenyl)-1,2,3,6-tetrahydro-pyridine (Table 2, entry 12)<smiles>FC(F)(F)c1ccc(C2=CCN(Cc3ccccc3)CC2)cc1</smiles>
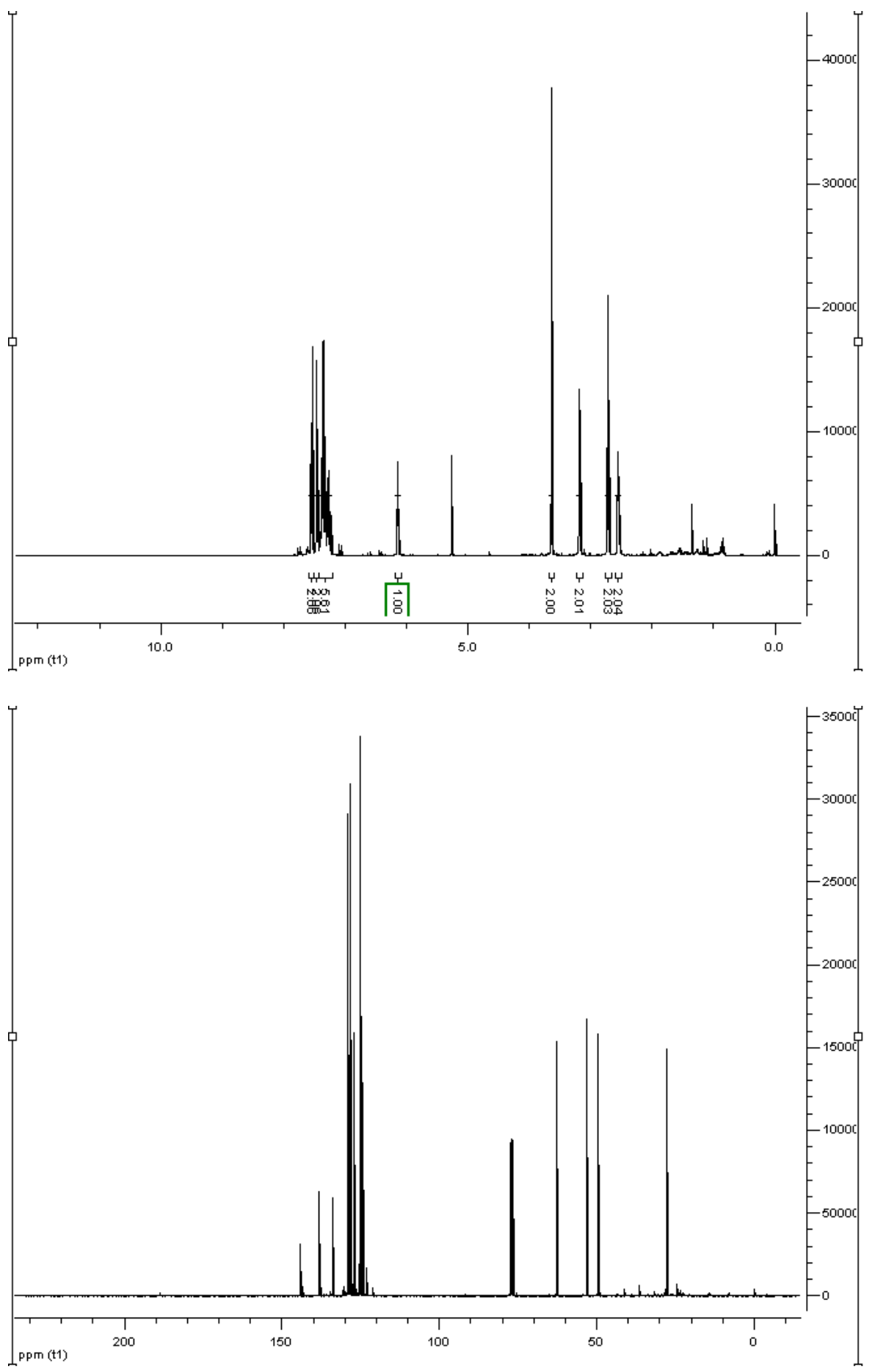
1-[4-(1-Benzyl-1,2,3,6-tetrahydro-pyridin-4-yl)-phenyl]-ethanone (Table 2, entry 13)<smiles>CC(=O)c1ccc(C2=CCN(Cc3ccccc3)CC2)cc1</smiles>
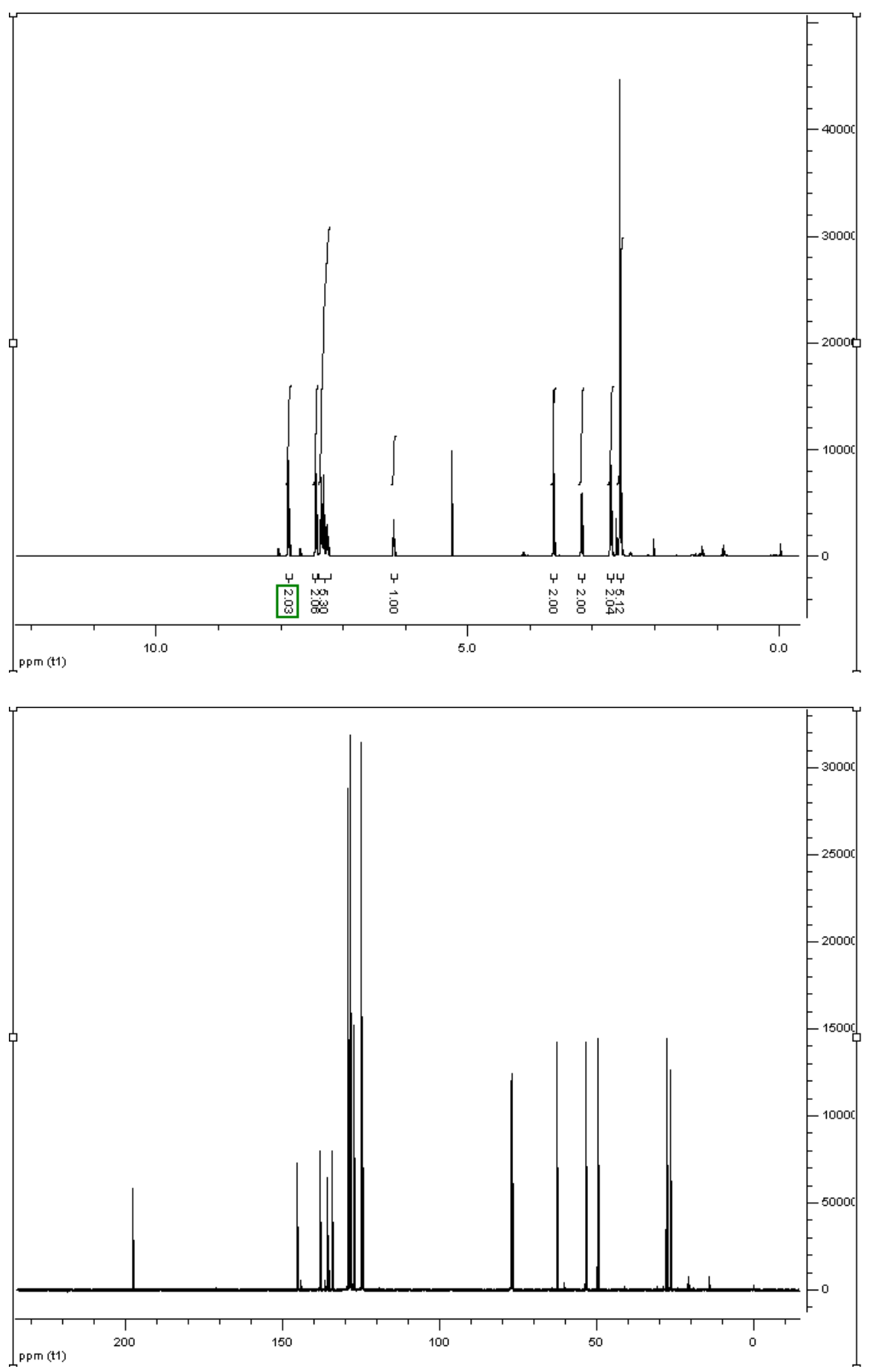
4-(1-Benzyl-1,2,3,6-tetrahydro-pyridin-4-yl)-benzoic acid methyl ester (Table 2, entry 14)<smiles>COC(=O)c1ccc(C2=CCN(Cc3ccccc3)CC2)cc1</smiles>
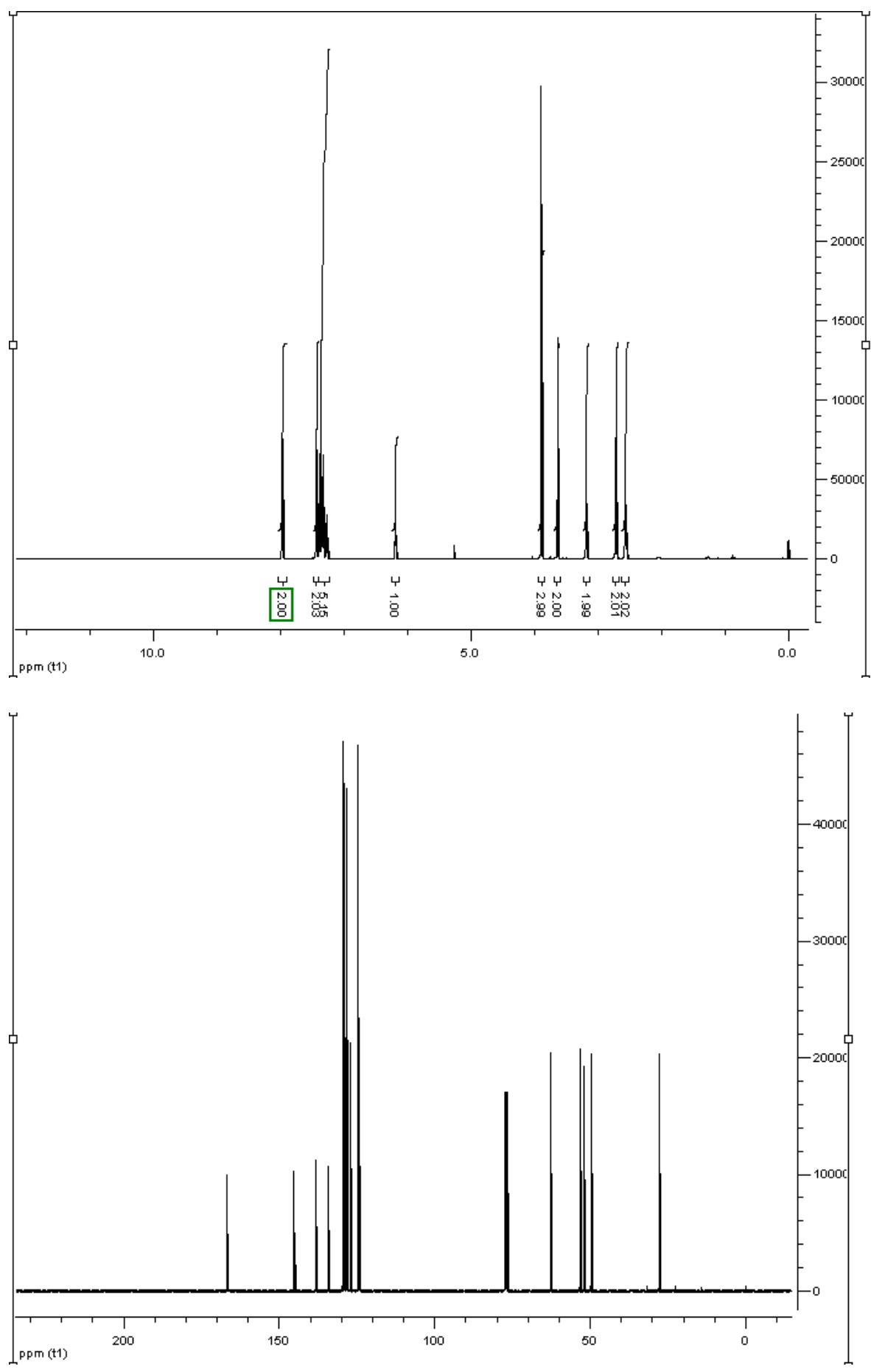
4-(1-Benzyl-1,2,3,6-tetrahydro-pyridin-4-yl)-benzonitrile (Table 2, entry 15)<smiles>N#Cc1ccc(C2=CCN(Cc3ccccc3)CC2)cc1</smiles>
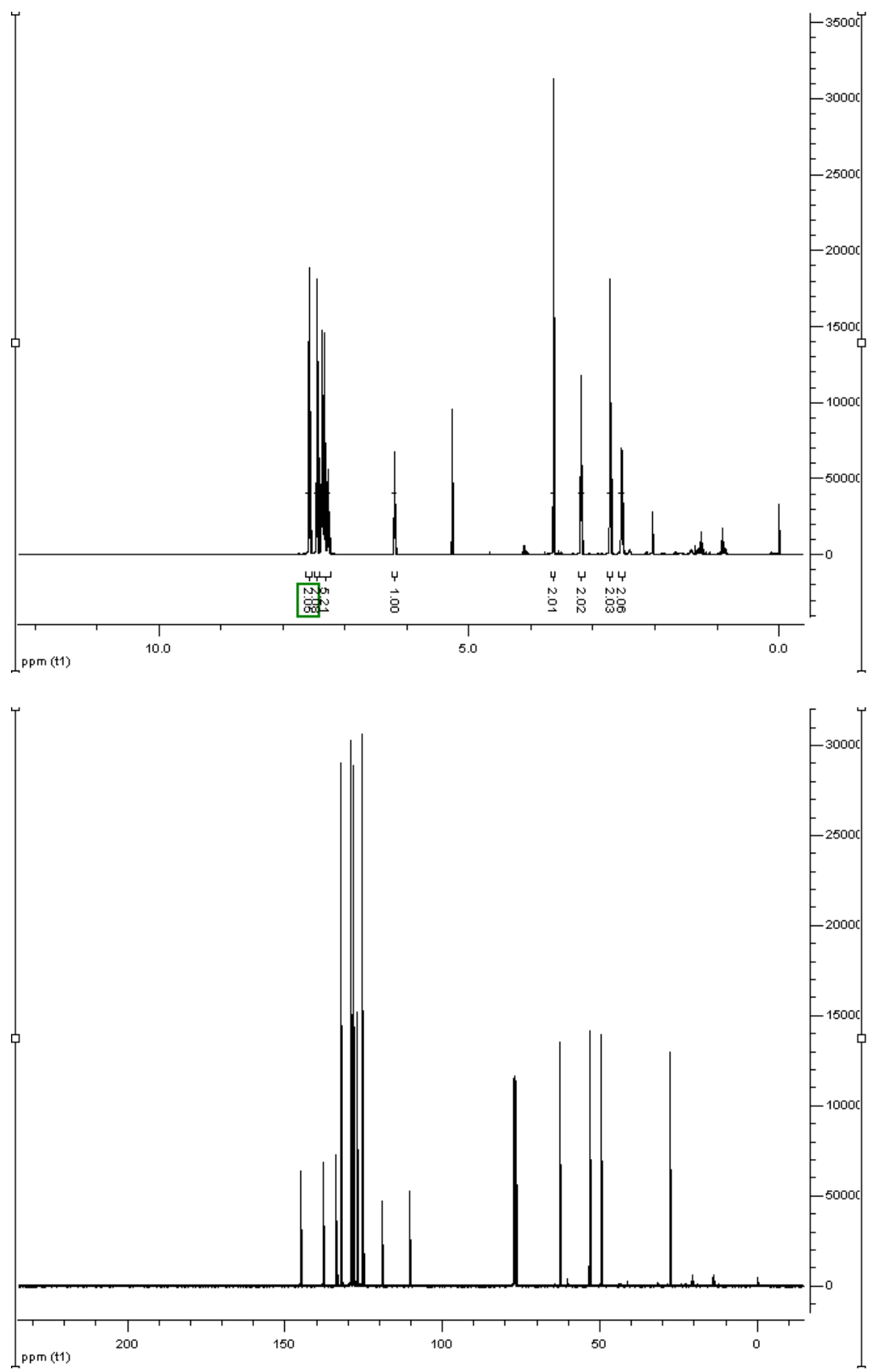
1-Benzyl-4-(4-bromo-phenyl)-1,2,3,6-tetrahydro-pyridine (Table 2, entry 16)<smiles>Brc1ccc(C2=CCN(Cc3ccccc3)CC2)cc1</smiles>
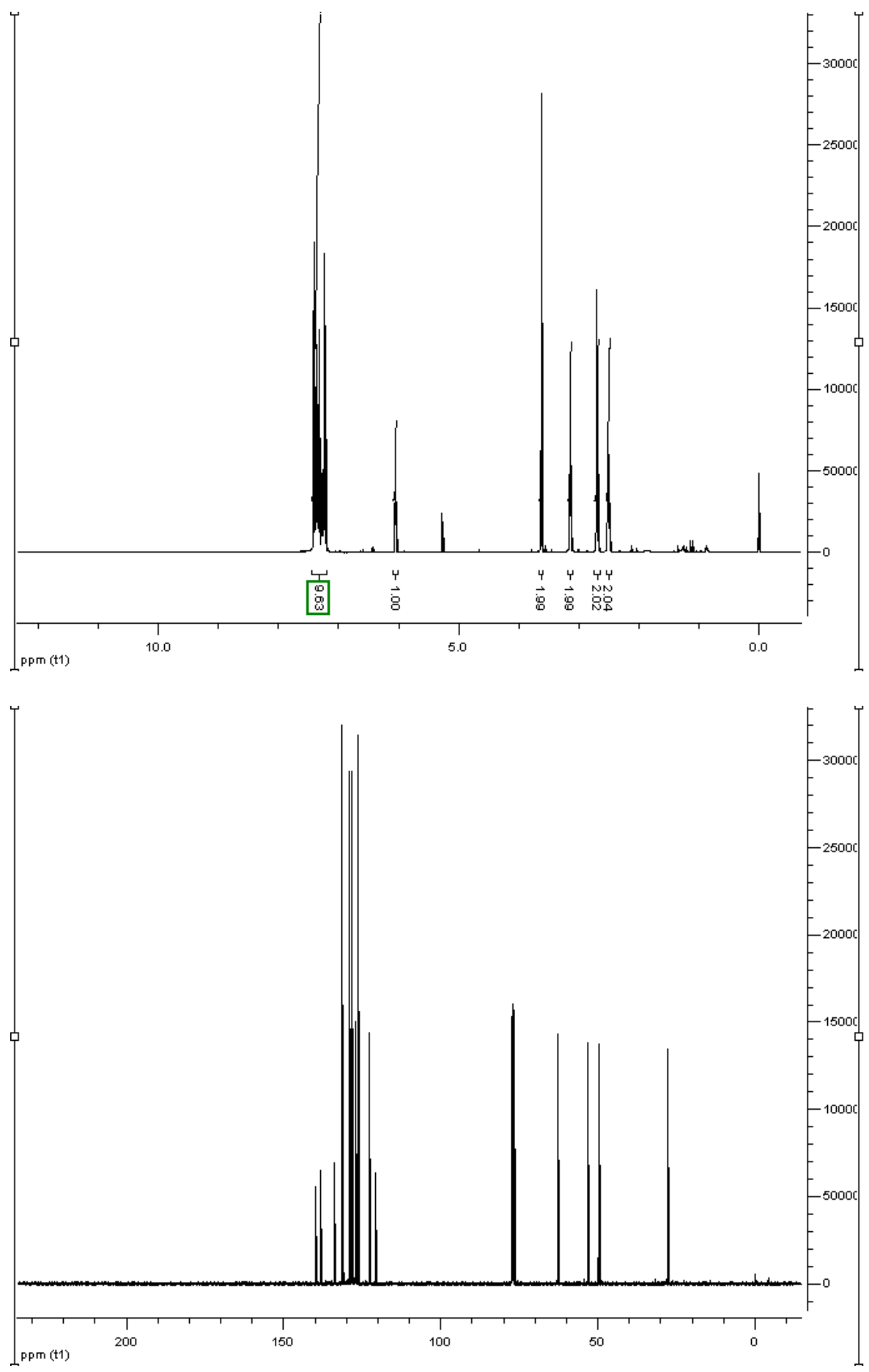
1-Benzyl-4-phenyl-1,2,3,6-tetrahydro-pyridine (Table 3, entry 4)<smiles>C1=C(c2ccccc2)CCN(Cc2ccccc2)C1</smiles>
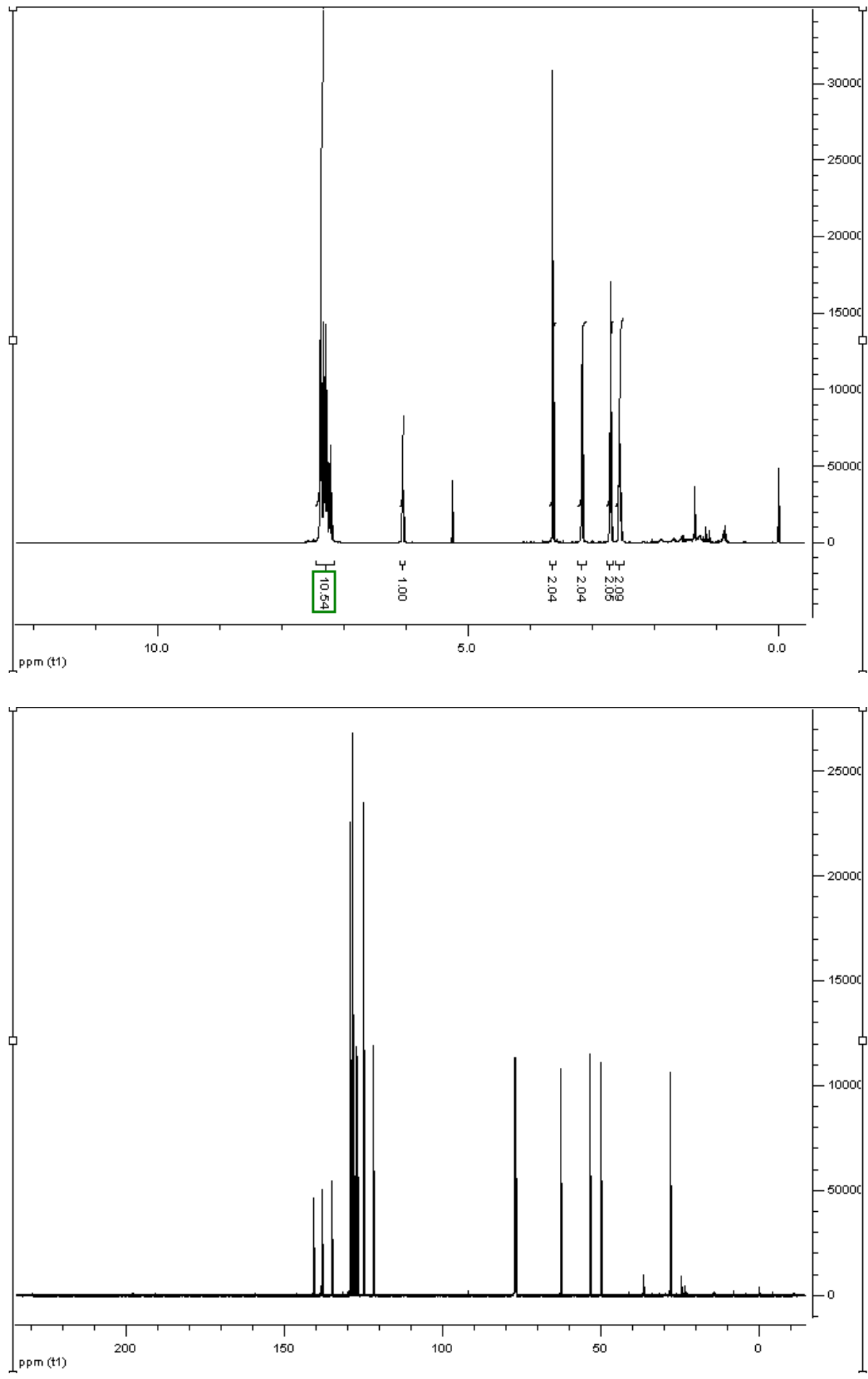
1-Benzyl-4-(4-trifluoromethyl-phenyl)-1,2,3,6-tetrahydro-pyridine (Table 3, entry 8)<smiles>FC(F)(F)c1ccc(C2=CCN(Cc3ccccc3)CC2)cc1</smiles>
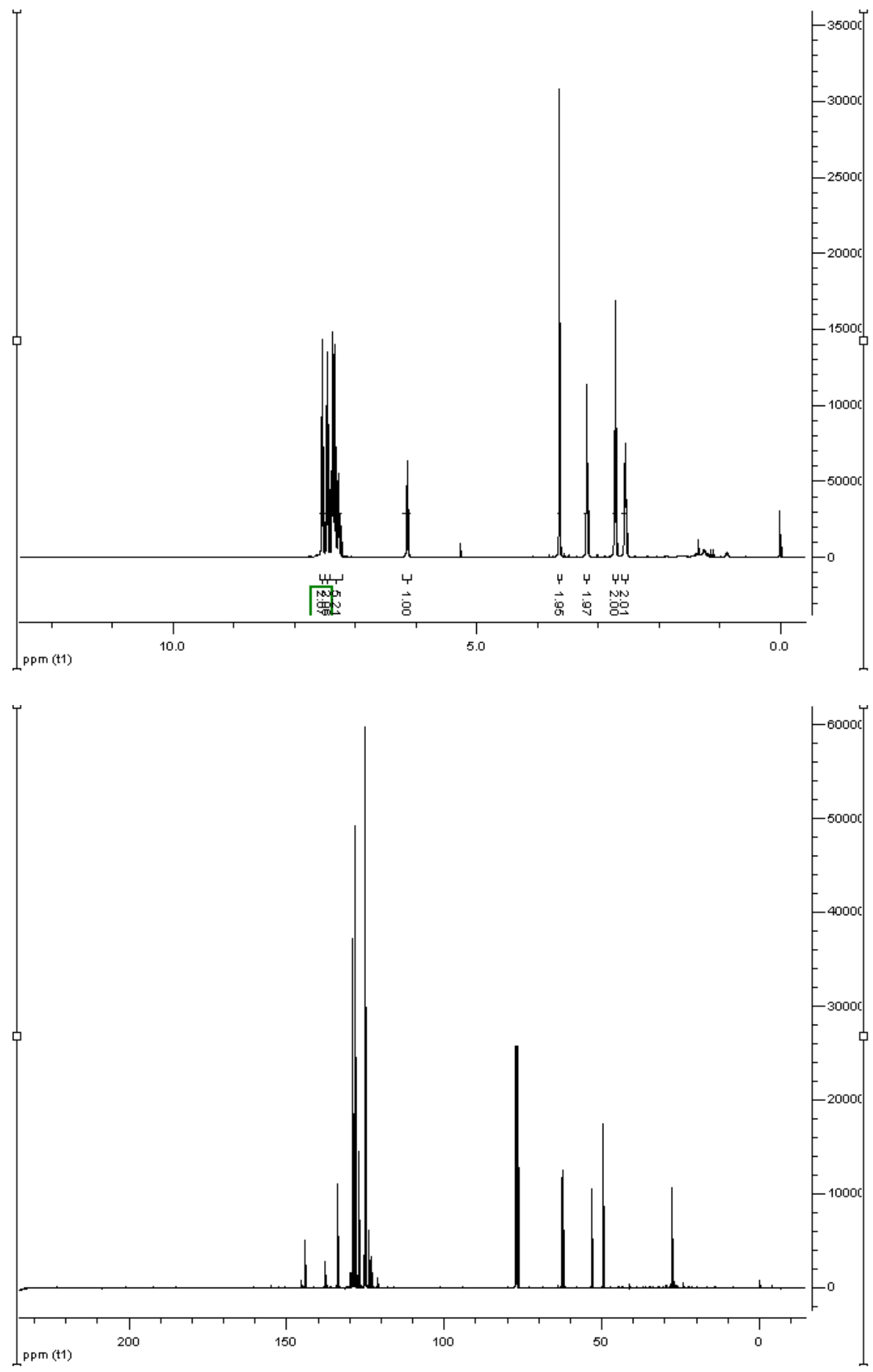
1-Benzyl-4-p-tolyl-1,2,3,6-tetrahydro-pyridine (Table 3, entry 9)<smiles>Cc1ccc(C2=CCN(Cc3ccccc3)CC2)cc1</smiles>
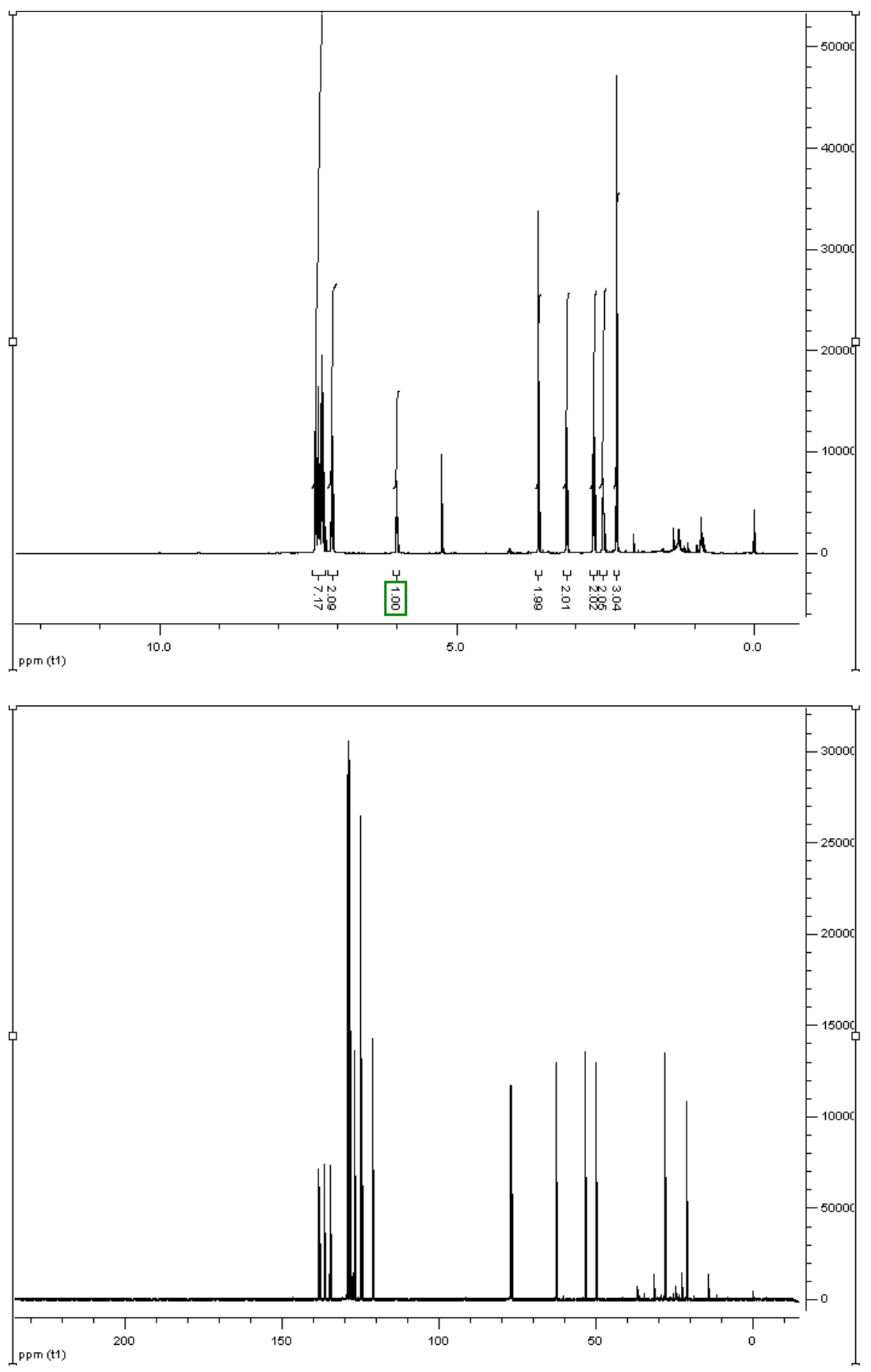
1-Benzyl-4-(4-methoxy-phenyl)-1,2,3,6-tetrahydro-pyridine (Table 3, entry 10)<smiles>COc1ccc(C2=CCN(Cc3ccccc3)CC2)cc1</smiles>
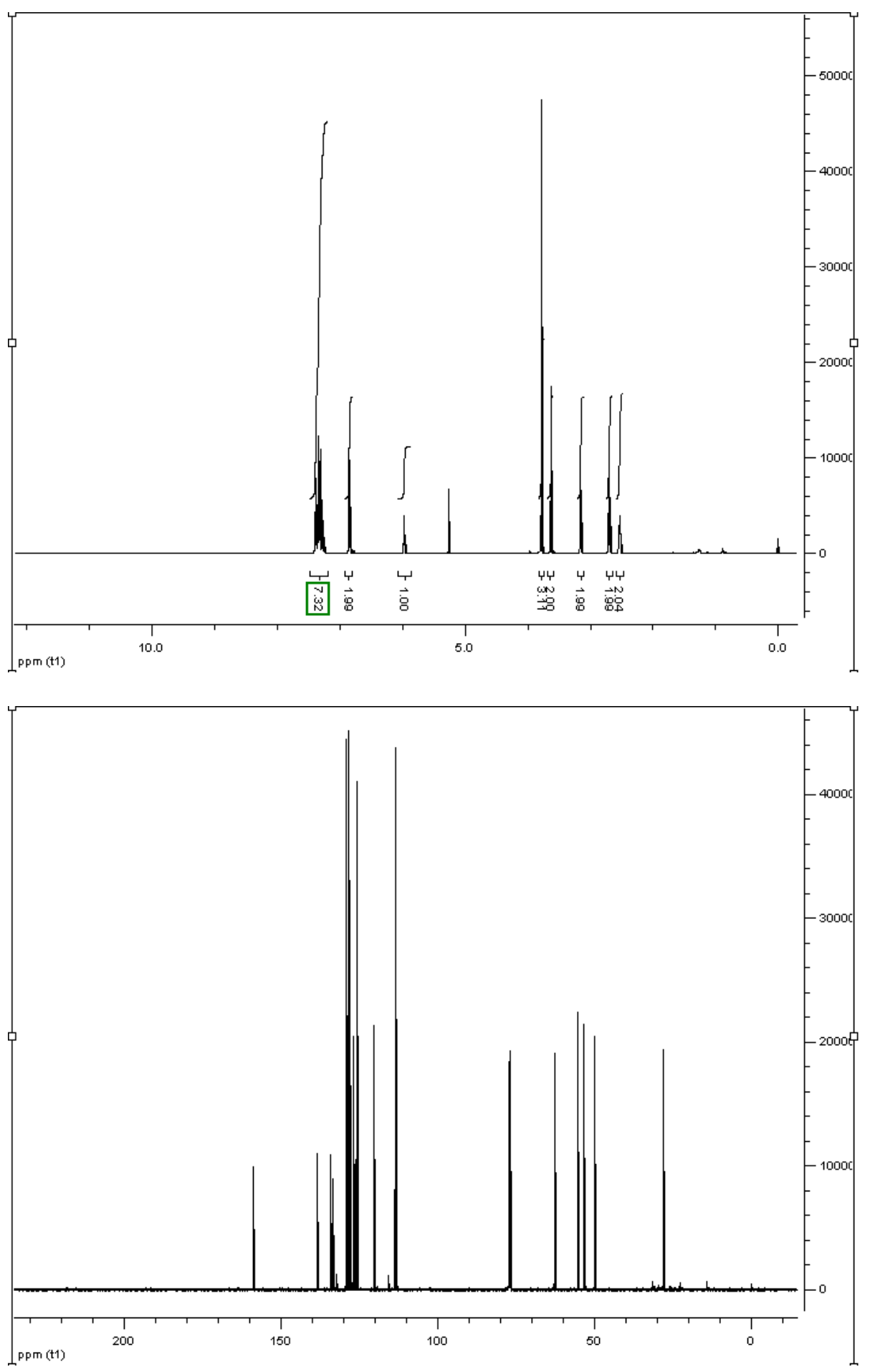
1-Benzyl-4-thiophen-3-yl-1,2,3,6-tetrahydro-pyridine (Table 3, entry 11)<smiles>C1=C(c2ccsc2)CCN(Cc2ccccc2)C1</smiles>
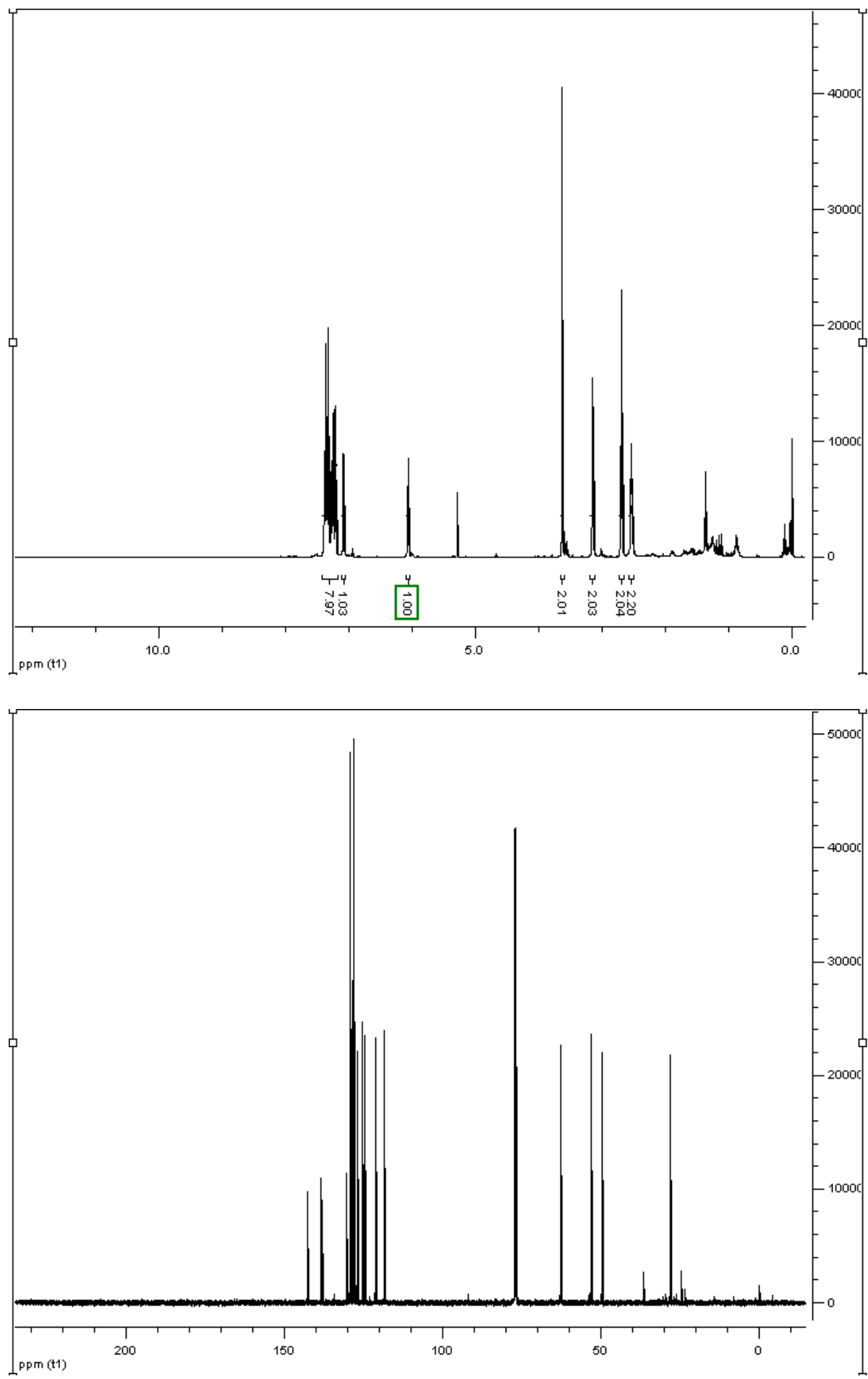
1-Benzyl-4-(4-fluoro-phenyl)-1,2,3,6-tetrahydro-pyridine (Table 3, entry 12)<smiles>Fc1ccc(C2=CCN(Cc3ccccc3)CC2)cc1</smiles>
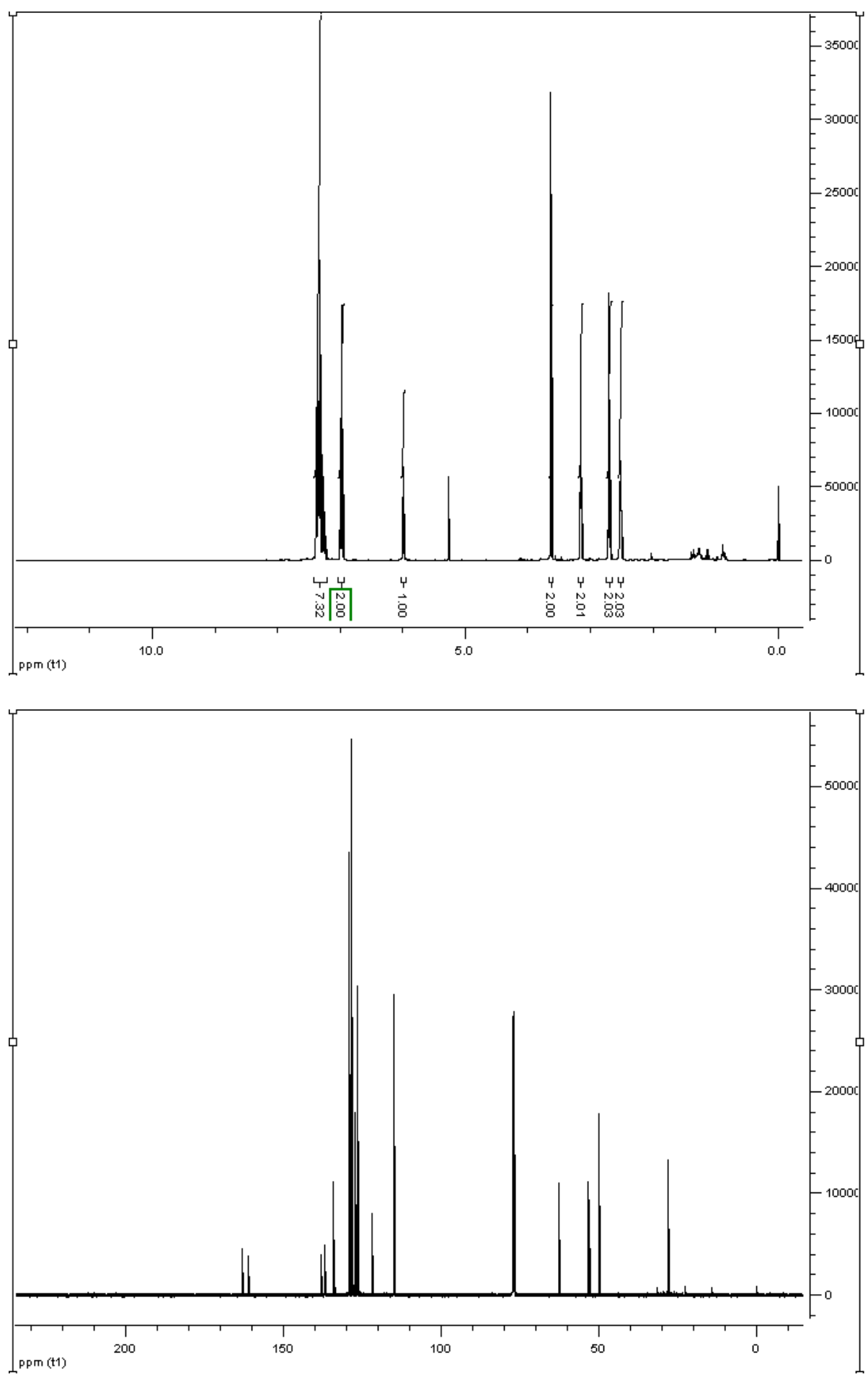
1-[4-(1-Benzyl-1,2,3,6-tetrahydro-pyridin-4-yl)-phenyl]-ethanone (Table 3, entry 13)<smiles>CC(=O)c1ccc(C2=CCN(Cc3ccccc3)CC2)cc1</smiles>
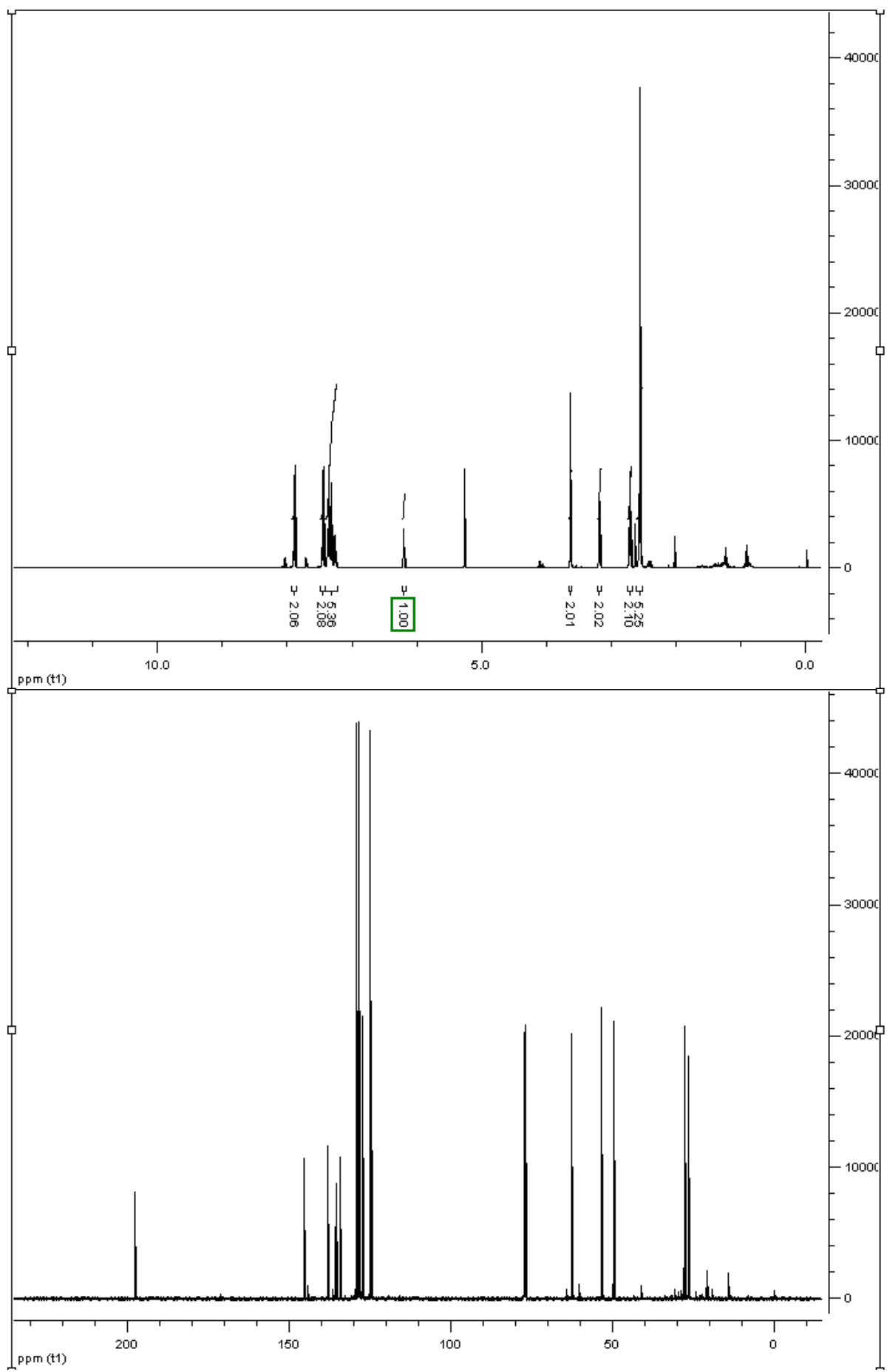
4-(1-Benzyl-1,2,3,6-tetrahydro-pyridin-4-yl)-benzoic acid methyl ester (Table 3, entry 14)<smiles>COC(=O)c1ccc(C2=CCN(Cc3ccccc3)CC2)cc1</smiles>
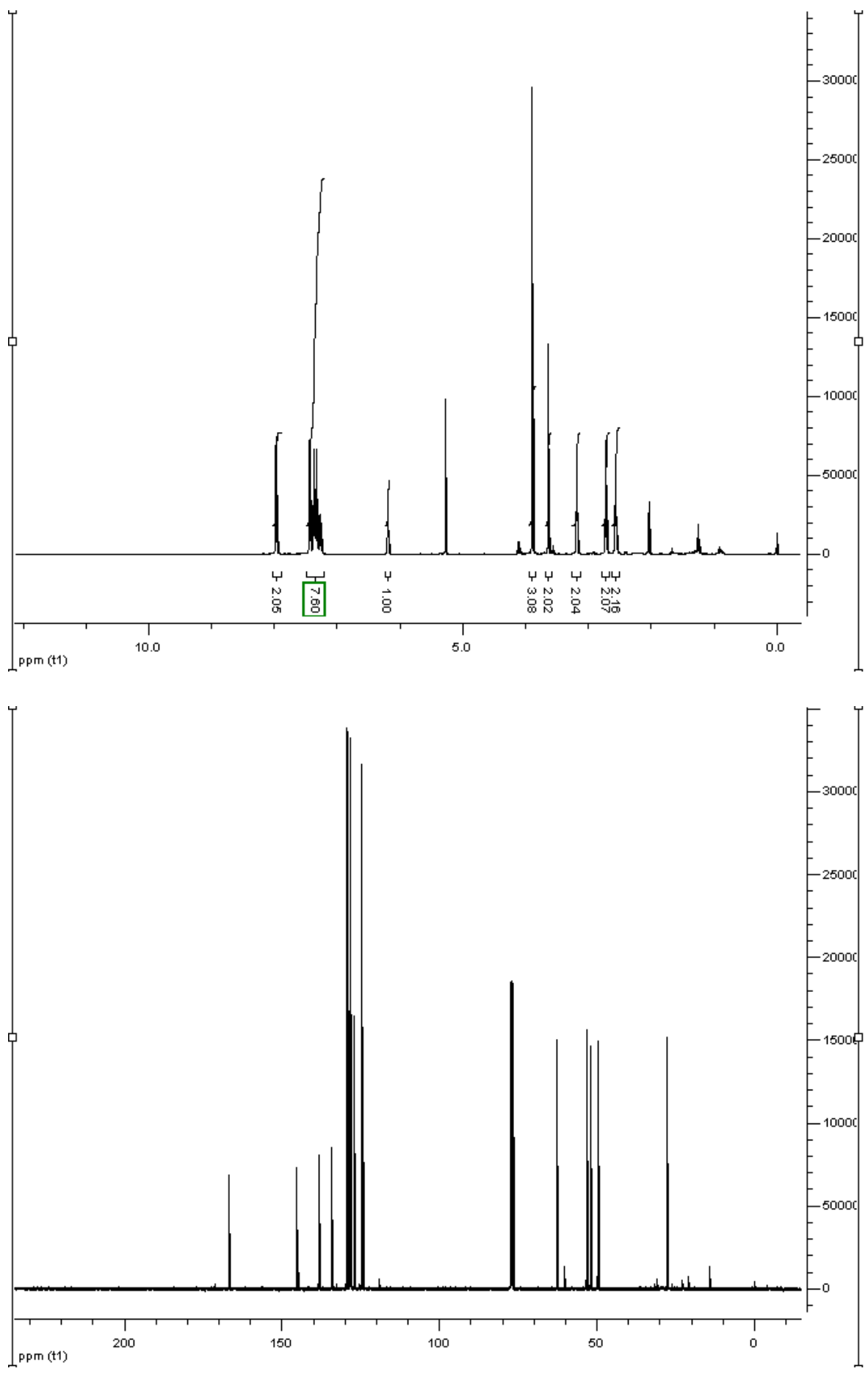
4-(1-Benzyl-1,2,3,6-tetrahydro-pyridin-4-yl)-benzonitrile (Table 3, entry 15)<smiles>N#Cc1ccc(C2=CCN(Cc3ccccc3)CC2)cc1</smiles>
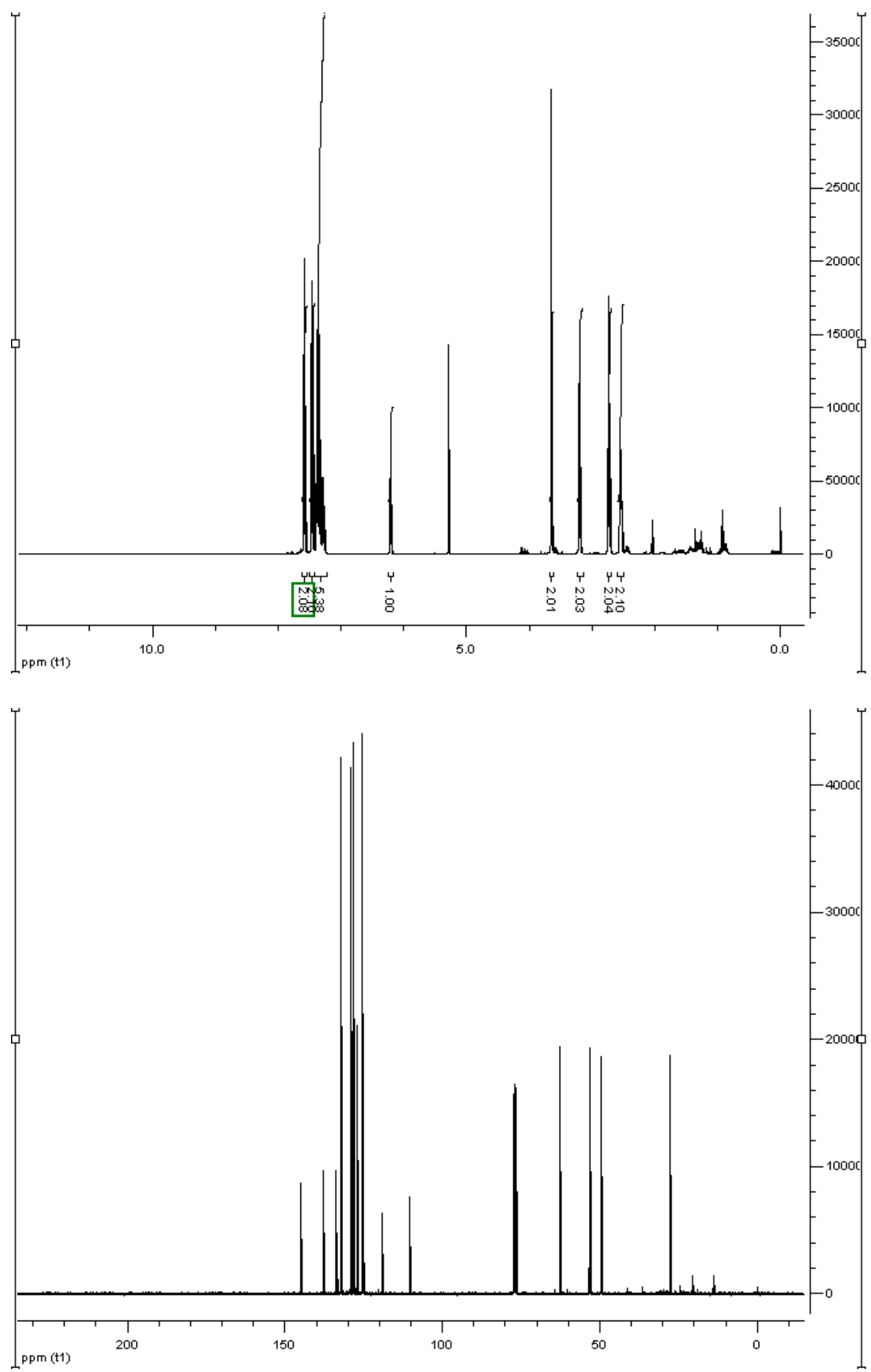